\title{
Stereocontrolled Total Synthesis of (+)-Isolaurenidificin and $(-)$-Bromlaurenidificin
}

\author{
Shoji Kobayashi,* Yutaka Hori, Ryo Yoneyama, Tomoki Tamura, \\ and Araki Masuyama
}

Department of Applied Chemistry, Faculty of Engineering, Osaka Institute of Technology, 5-16-1 Ohmiya, Asahi-ku, Osaka 535-8585, Japan

shoji.kobayashi@oit.ac.jp

Supporting Information 


\section{TABLE OF CONTENTS}

1. Comparisons of ${ }^{1} \mathrm{H}$ and ${ }^{13} \mathrm{C}$ NMR chemical shifts for natural and synthetic compounds .........S3

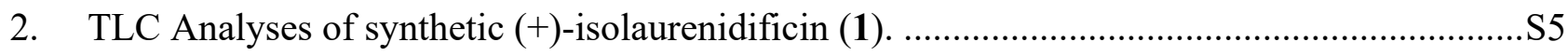

3. Comparisons of ${ }^{1} \mathrm{H}$ NMR spectra of selected epimers..........................................................S5

4. Structures of intermediates that are not depicted in Schemes............................................... 7

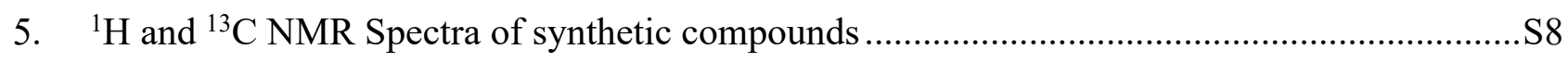




\section{Comparisons of ${ }^{1} \mathrm{H}$ and ${ }^{13} \mathrm{C}$ NMR chemical shifts for natural and synthetic compounds}

Table S1. Comparisons of ${ }^{1} \mathrm{H}$ and ${ }^{13} \mathrm{C}$ NMR chemical shifts for natural ${ }^{1}$ and synthetic isolaurenidificin (1) in $\mathrm{CDCl}_{3}{ }^{e}$

\begin{tabular}{|c|c|c|c|c|c|c|}
\hline \multirow[b]{2}{*}{ position } & \multicolumn{3}{|c|}{$\begin{array}{c}\text { isolaurenidificin (1) } \\
\text { (natural product) }\end{array}$} & \multicolumn{3}{|c|}{ 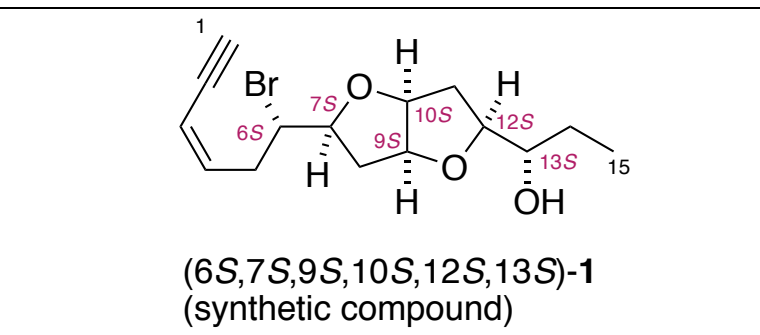 } \\
\hline & $\delta\left\{{ }^{1} \mathrm{H}\right\}^{a}$ & $J / \mathrm{Hz}$ & $\delta\left\{{ }^{13} \mathrm{C}\right\}^{b}$ & $\delta\left\{{ }^{1} \mathrm{H}\right\}^{c}$ & $J / \mathrm{Hz}$ & $\delta\left\{{ }^{13} \mathrm{C}\right\}^{d}$ \\
\hline 1 & $3.15(d)$ & 1.7 & 82.8 & $3.15(\mathrm{dd})$ & $2.4,0.8$ & 82.9 \\
\hline 2 & & & 79.9 & & & 80.0 \\
\hline 3 & 5.63 (ddd) & $11.1,3.4,1.7$ & 111.0 & $5.62(\mathrm{ddt})$ & $11,2.4,1.5$ & 111.1 \\
\hline 4 & 6.18 (ddd) & $11.1,10.2,7.7$ & 141.1 & $6.19(\mathrm{dtd})$ & $11,6.8,0.8$ & 141.2 \\
\hline \multirow[t]{2}{*}{5} & $\begin{array}{l}2.96 \\
\text { (dddd) }\end{array}$ & $\begin{array}{lll}15.3, & 8.5, & 7.0 \\
1.7\end{array}$ & 35.5 & $3.00-2.86(\mathrm{~m})$ & & 35.7 \\
\hline & $2.90(\mathrm{~m})$ & & & & & \\
\hline 6 & 4.15 (ddd) & $10.2,8.5,4.3$ & 55.7 & 4.15 (ddd) & $8.2,6.9,4.4$ & 55.8 \\
\hline 7 & $4.08(\mathrm{dd})$ & $14.5,7.7$ & 83.6 & 4.08 (ddd) & $7.6,7.3,6.9$ & 83.7 \\
\hline \multirow[t]{2}{*}{8} & 2.32 (ddd) & $13.6,7.7,6.8$ & 36.8 & 2.33 (ddd) & $14,7.3,6.1$ & 37.0 \\
\hline & $2.02(\mathrm{~m})$ & & & $2.06-1.97(\mathrm{~m})$ & & \\
\hline 9 & 4.55 (ddd) & $6.0,4.3,1.7$ & 84.3 & 4.55 (ddd) & $6.1,4.1,1.7$ & 84.5 \\
\hline 10 & $4.53(\mathrm{ddd})$ & $6.0,4.3,1.7$ & 85.0 & 4.53 (ddd) & $6.1,4.1,1.7$ & 85.1 \\
\hline \multirow[t]{2}{*}{11} & 2.22 (ddd) & $13.6,7.7,6.8$ & 34.9 & 2.22 (ddd) & $14,7.8,6.1$ & 35.0 \\
\hline & $2.02(\mathrm{~m})$ & & & $2.06-1.97(\mathrm{~m})$ & & \\
\hline 12 & 3.88 (ddd) & $14.7,8.5,6.8$ & 84.3 & $3.89(\mathrm{dt})$ & $7.8,6.5$ & 84.4 \\
\hline 13 & 3.54 (ddd) & $11.1,6.8,4.3$ & 75.2 & 3.54 (ddd) & $8.3,6.5,4.2$ & 75.3 \\
\hline \multirow[t]{2}{*}{14} & $1.51(\mathrm{~m})$ & & 26.5 & $1.56-1.38(\mathrm{~m})$ & & 26.6 \\
\hline & $1.45(\mathrm{~m})$ & & & & & \\
\hline 15 & $1.00(\mathrm{t})$ & 6.8 & 10.1 & $1.00(\mathrm{t})$ & 7.4 & 10.2 \\
\hline
\end{tabular}

${ }^{a}$ Measured at $850 \mathrm{MHz}$. No mention on the reference peak from literature. ${ }^{b}$ Measured at 212.5 MHz. No mention on the reference peak from literature. ${ }^{c}$ Measured at $400 \mathrm{MHz}$. Chemical shifts are referenced to the solvent peak at $7.26 \mathrm{ppm} .{ }^{d}$ Measured at $100 \mathrm{MHz}$. Chemical shifts are referenced to the solvent peak at $77.16 \mathrm{ppm} .{ }^{e}$ Suspected multiplicity and coupling constants are highlighted in blue. Unconvincing data for which no expanded spectra are disclosed are given in red. 
Table S2. Comparisons of ${ }^{1} \mathrm{H}$ and ${ }^{13} \mathrm{C}$ NMR chemical shifts for natural ${ }^{1}$ and synthetic bromlaurenidificin (2) in $\mathrm{C}_{6} \mathrm{D}_{6}{ }^{e}$

\begin{tabular}{|c|c|c|c|c|c|c|}
\hline \multirow[b]{2}{*}{ position } & \multicolumn{3}{|c|}{ bromlaurenidificin (2) } & \multicolumn{3}{|c|}{ 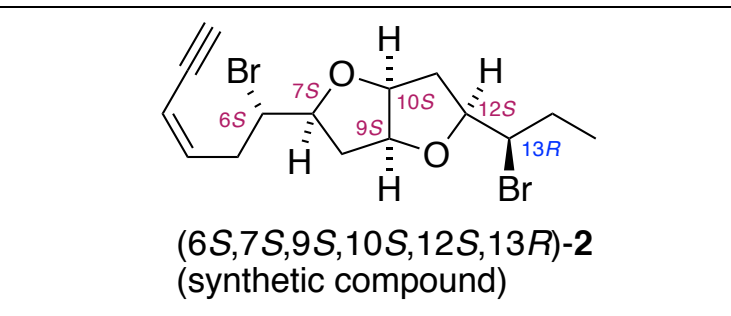 } \\
\hline & $\delta\left\{{ }^{1} \mathrm{H}\right\}^{a}$ & $J / \mathrm{Hz}$ & $\delta\left\{{ }^{13} \mathrm{C}\right\}^{b}$ & $\delta\left\{{ }^{1} \mathrm{H}\right\}^{c}$ & $J / \mathrm{Hz}$ & $\delta\left\{{ }^{13} \mathrm{C}\right\}^{d}$ \\
\hline 1 & $2.76(d)$ & 1.7 & 82.8 & $2.76(\mathrm{dd})$ & $2.3,0.9$ & 83.1 \\
\hline 2 & & & 79.8 & & & 80.2 \\
\hline 3 & $5.35(\mathrm{ddd})$ & $11.1,2.6,1.7$ & 110.6 & $5.36(\mathrm{ddt})$ & $11,2.3,1.4$ & 111.0 \\
\hline 4 & 5.85 (ddd) & $11.9,11.1,6.8$ & 141.4 & $5.86(\mathrm{dtd})$ & $11,7.1,0.9$ & 141.7 \\
\hline \multirow[t]{2}{*}{5} & $2.87(\mathrm{~m})$ & & 35.4 & $2.92-2.78(\mathrm{~m})$ & & 35.8 \\
\hline & $2.83(\mathrm{~m})$ & & & & & \\
\hline 6 & $3.83(\mathrm{~m})$ & & 55.0 & $3.85-3.81(\mathrm{~m})$ & & 55.4 \\
\hline 7 & 3.56 (ddd) & $12.8,7.7,5.1$ & 82.7 & 3.56 (ddd) & $8.2,7.1,5.0$ & 83.0 \\
\hline \multirow[t]{2}{*}{8} & 1.85 (ddd) & $13.6,6.0,1.7$ & 36.9 & $1.85(\mathrm{ddd})$ & $14,8.2,2.2$ & 37.3 \\
\hline & $1.66(\mathrm{ddd})$ & $13.6,6.8,1.7$ & & $1.66(\mathrm{ddd})$ & $14,7.1,6.6$ & \\
\hline 9 & 3.99 (ddd) & $6.0,3.4,1.7$ & 84.9 & 4.00 (ddd) & $6.6,4.3,2.2$ & 85.3 \\
\hline 10 & $3.82(\mathrm{~m})$ & & 84.1 & $3.85-3.81(\mathrm{~m})$ & & 84.4 \\
\hline \multirow[t]{2}{*}{11} & $2.28(\mathrm{dd})$ & $13.6,7.7$ & 37.5 & $2.29(\mathrm{ddd})$ & $14,5.4,1.5$ & 37.9 \\
\hline & 1.91 (ddd) & $13.6,7.7,6.0$ & & $1.92(\mathrm{ddd})$ & $14,7.8,6.2$ & \\
\hline 12 & 3.93 (ddd) & $9.4,8.5,6.0$ & 83.6 & 3.94 (ddd) & $9.2,7.8,5.4$ & 83.9 \\
\hline 13 & 4.24 (ddd) & $11.9,9.4,2.6$ & 61.4 & $4.25(\mathrm{td})$ & $9.2,2.9$ & 61.8 \\
\hline \multirow[t]{2}{*}{14} & $\begin{array}{l}2.15 \\
\text { (dddd) }\end{array}$ & $14.5,10.2,7.7,2.6$ & 28.4 & $2.16(\mathrm{dqd})$ & $15,7.3,2.9$ & 28.7 \\
\hline & $1.76(\mathrm{ddq})$ & $14.5,9.4,7.7$ & & $1.83-1.71(\mathrm{~m})$ & & \\
\hline 15 & $1.04(\mathrm{t})$ & 7.7 & 11.1 & $1.06(\mathrm{t})$ & 7.4 & 11.6 \\
\hline
\end{tabular}

${ }^{a}$ Measured at $850 \mathrm{MHz}$. No mention on the reference peak from literature. ${ }^{b}$ Measured at 212.5 MHz. No mention on the reference peak from literature. ${ }^{c}$ Measured at $400 \mathrm{MHz}$. Chemical shifts are referenced to the solvent peak at $7.16 \mathrm{ppm} .{ }^{d}$ Measured at $100 \mathrm{MHz}$. Chemical shifts are referenced to the solvent peak at $128.06 \mathrm{ppm} .{ }^{e}$ Suspected multiplicity and coupling constants are highlighted in blue. 
2. TLC Analyses of synthetic (+)-isolaurenidificin (1).

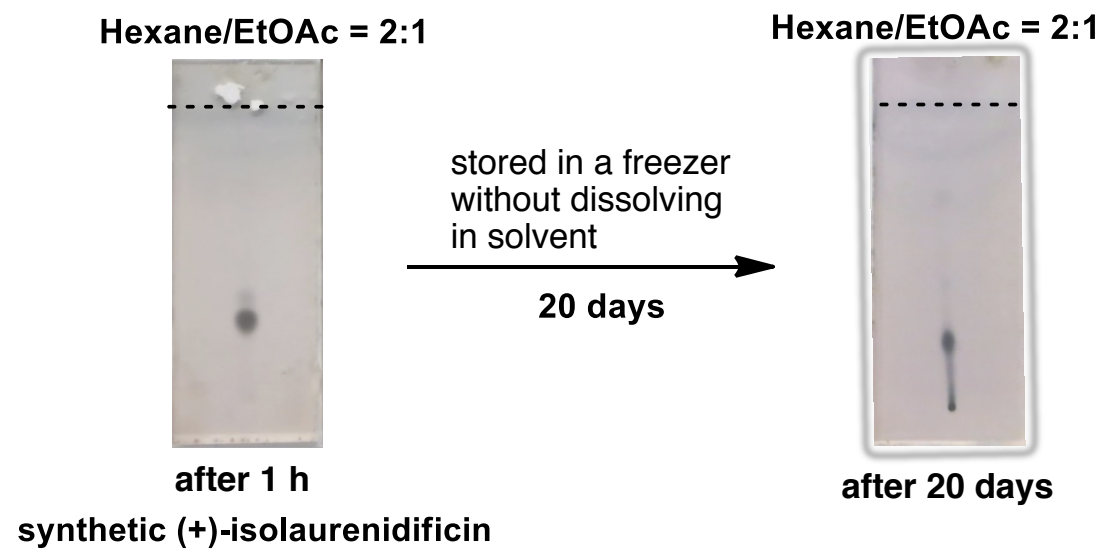

Figure S1. TLC analyses of synthetic (+)-isolaurenidificin (1). The compound was stored in a freezer at $-30{ }^{\circ} \mathrm{C}$ without dissolving in solvent for 20 days. TLC analyses were carried out after dissolving in DCM. A mixed solvent consisting of $n$-hexane and EtOAc $(2: 1 \mathrm{v} / \mathrm{v})$ was used as a developing solvent. TLC plates were stained by ethanol solution containing $p$-anisaldeyde, $\mathrm{AcOH}$, and $\mathrm{H}_{2} \mathrm{SO}_{4}$, and heated on a hot plate for a few seconds.

3. Comparisons of ${ }^{1} \mathrm{H}$ NMR spectra of selected epimers.

(a) ${ }^{1} \mathrm{H}$ NMR $\left(400 \mathrm{MHz}, \mathrm{CDCl}_{3}\right)$

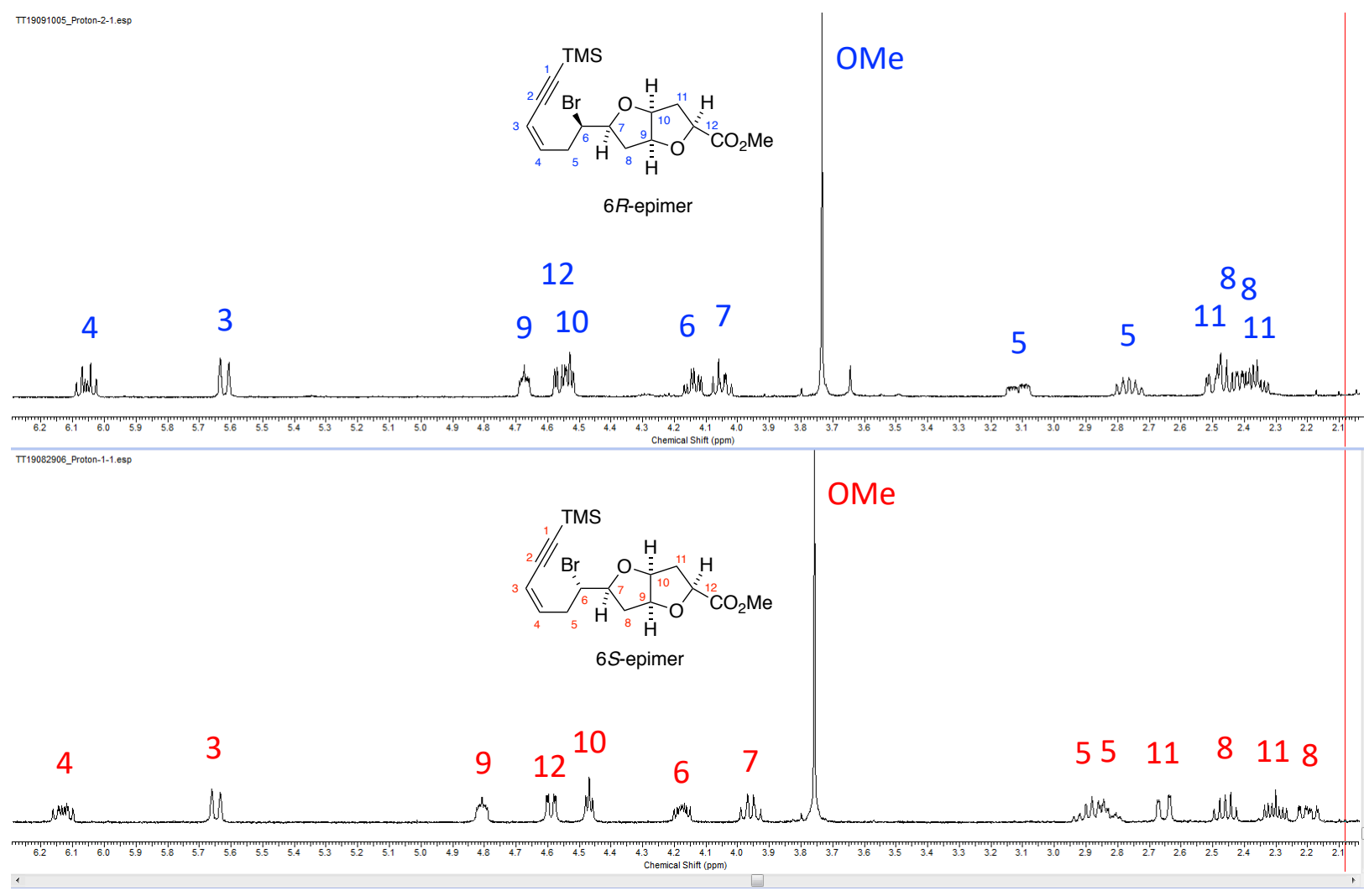

Figure S2. Comparisons of ${ }^{1} \mathrm{H}$ NMR spectra of epimers synthesized in our laboratory. Peak assignments were made on the basis of ${ }^{1} \mathrm{H}-{ }^{1} \mathrm{H}$ COSY spectra. 
(b) ${ }^{1} \mathrm{H}$ NMR $\left(400 \mathrm{MHz}, \mathrm{CDCl}_{3}\right)$

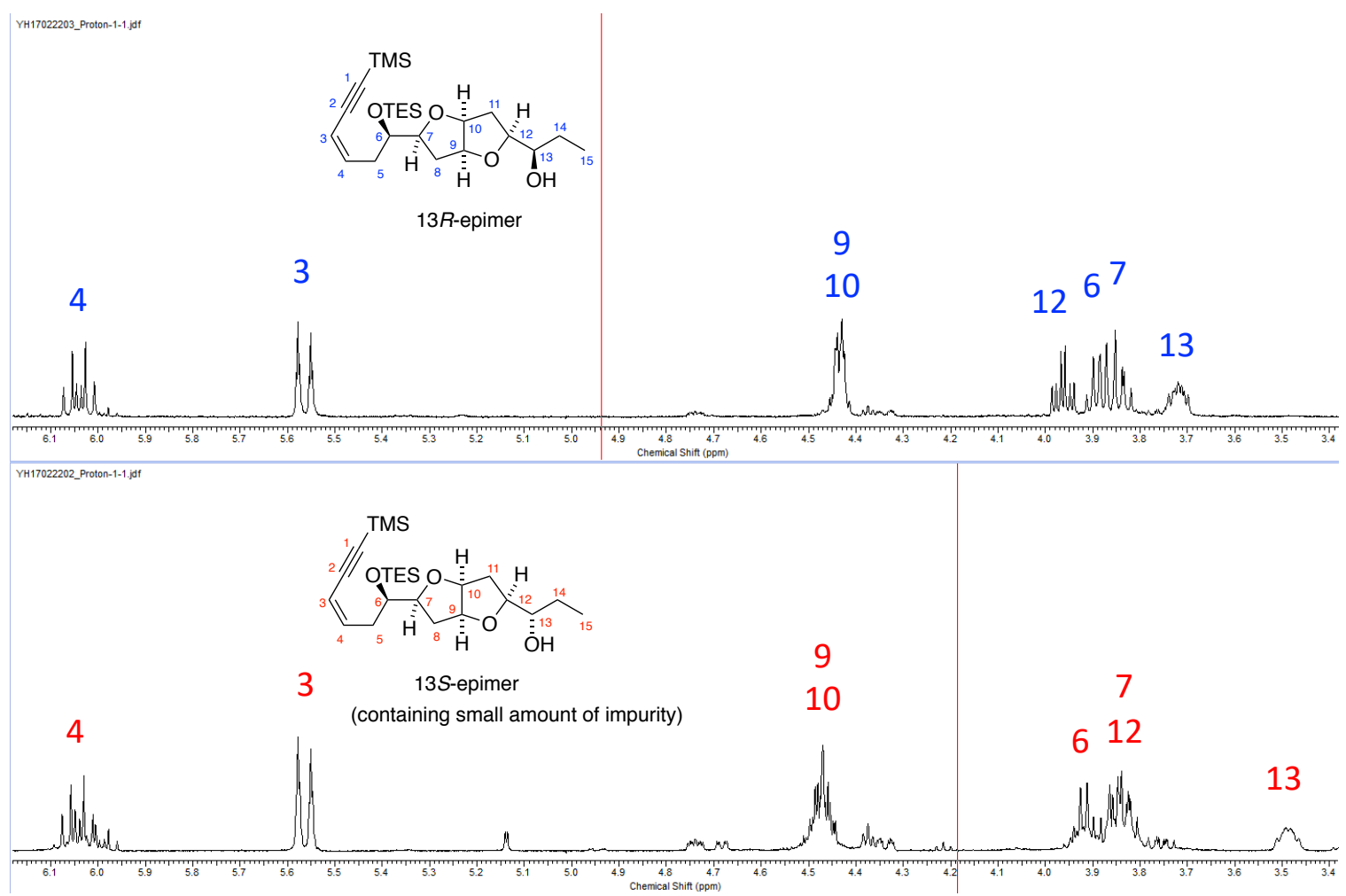

(c) ${ }^{1} \mathrm{H}$ NMR $\left(400 \mathrm{MHz}, \mathrm{CDCl}_{3}\right)$

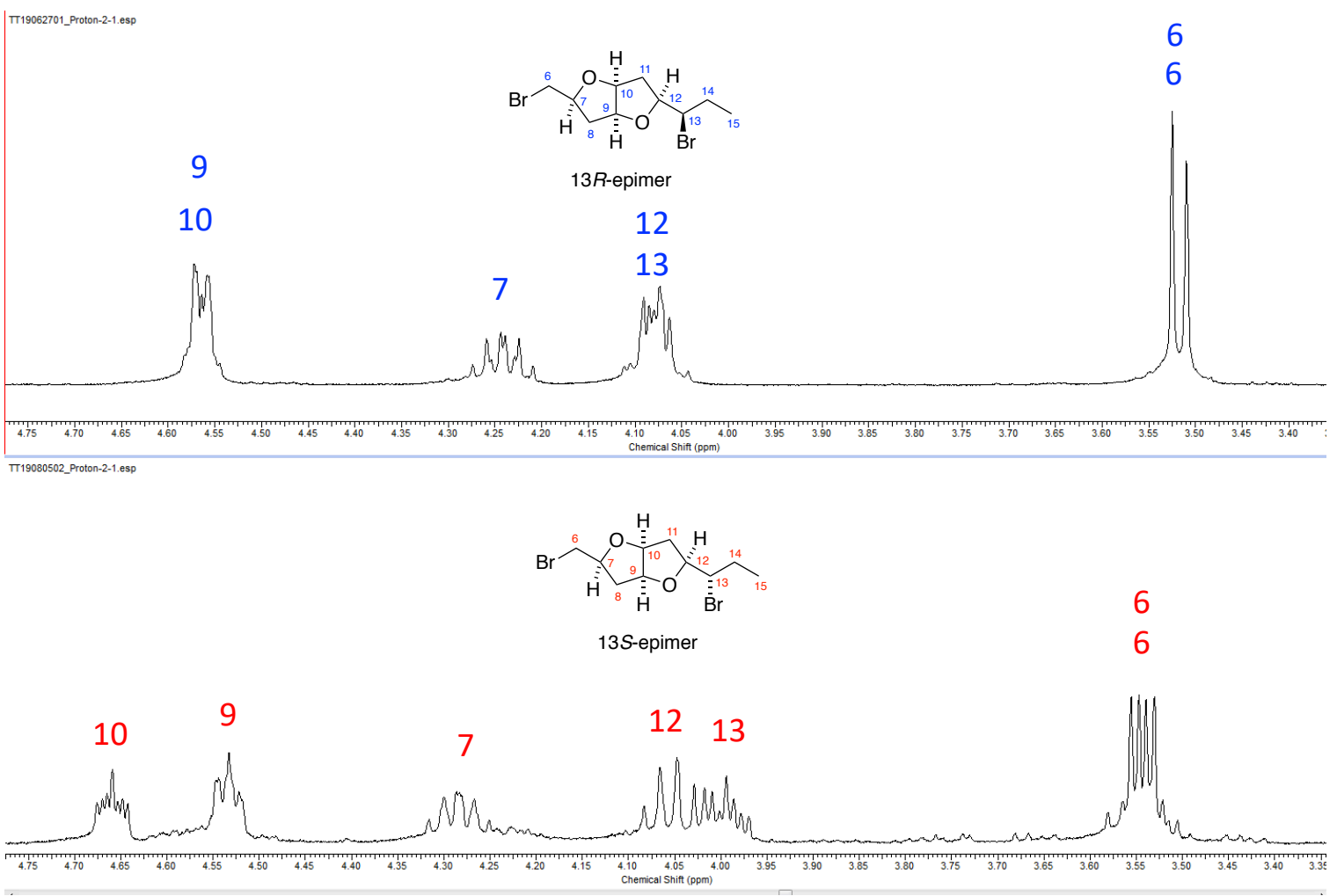

Figure S2 (continued). Comparisons of ${ }^{1} \mathrm{H}$ NMR spectra of epimers synthesized in our laboratory. Peak assignments were made on the basis of ${ }^{1} \mathrm{H}-{ }^{1} \mathrm{H}$ COSY spectra. 
4. Structures of intermediates that are not depicted in Schemes

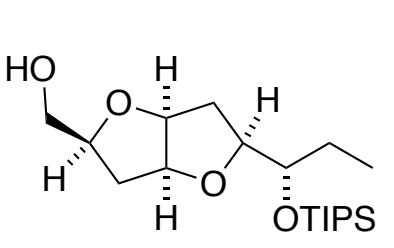

S1

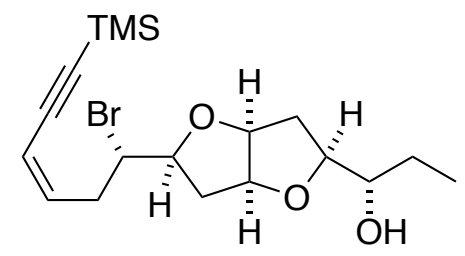

S2

\section{Reference}

(1) Bawakid, N.; Alarif, W.; Alburae, N.; Alorfi, H.; Al-Footy, K.; Al-Lihaibi, S.; Ghandourah, M. Molecules 2017, 22, 807. 
5. ${ }^{1} \mathrm{H}$ and ${ }^{13} \mathrm{C}$ NMR Spectra of synthetic compounds 
2019/04/19 10:06:43

\begin{tabular}{|c|c|c|c|c|c|c|c|c|}
\hline Acquisition Time (sec) & 2.1863 & Comment & single_pulse & \multicolumn{3}{|c|}{22 Sep 2017 14:13:39 } & Date Stamp & 22 Sep 2017 \\
\hline File Name & \multicolumn{4}{|c|}{ 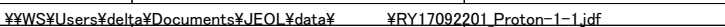 } & Frequency $(\mathrm{MHz})$ & 399.78 & Nucleus & $1 \mathrm{H}$ \\
\hline Number of Transients & 8 & Origin & ECA & Original Points Count & Ouner & delta & Points Count & 32768 \\
\hline Pulse Sequence & proton.jx & Solvent & CHLOROFO & & Spectrum Offset $(\mathrm{Hz})$ & 2003.4905 & Sweep Width $(\mathrm{Hz})$ & 7494.00 \\
\hline
\end{tabular}

12S-6_RY17092201_Proton-1-1.esp

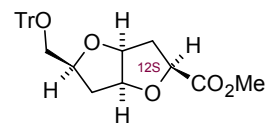

(12)-

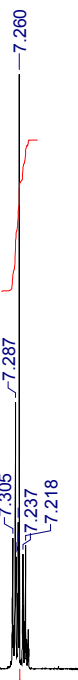

$\begin{array}{lllll}1.031 .031 .031 .04 & 3.131 .041 .02 & 1.04 & 2.18 & 1.07\end{array}$

6.7812 .44

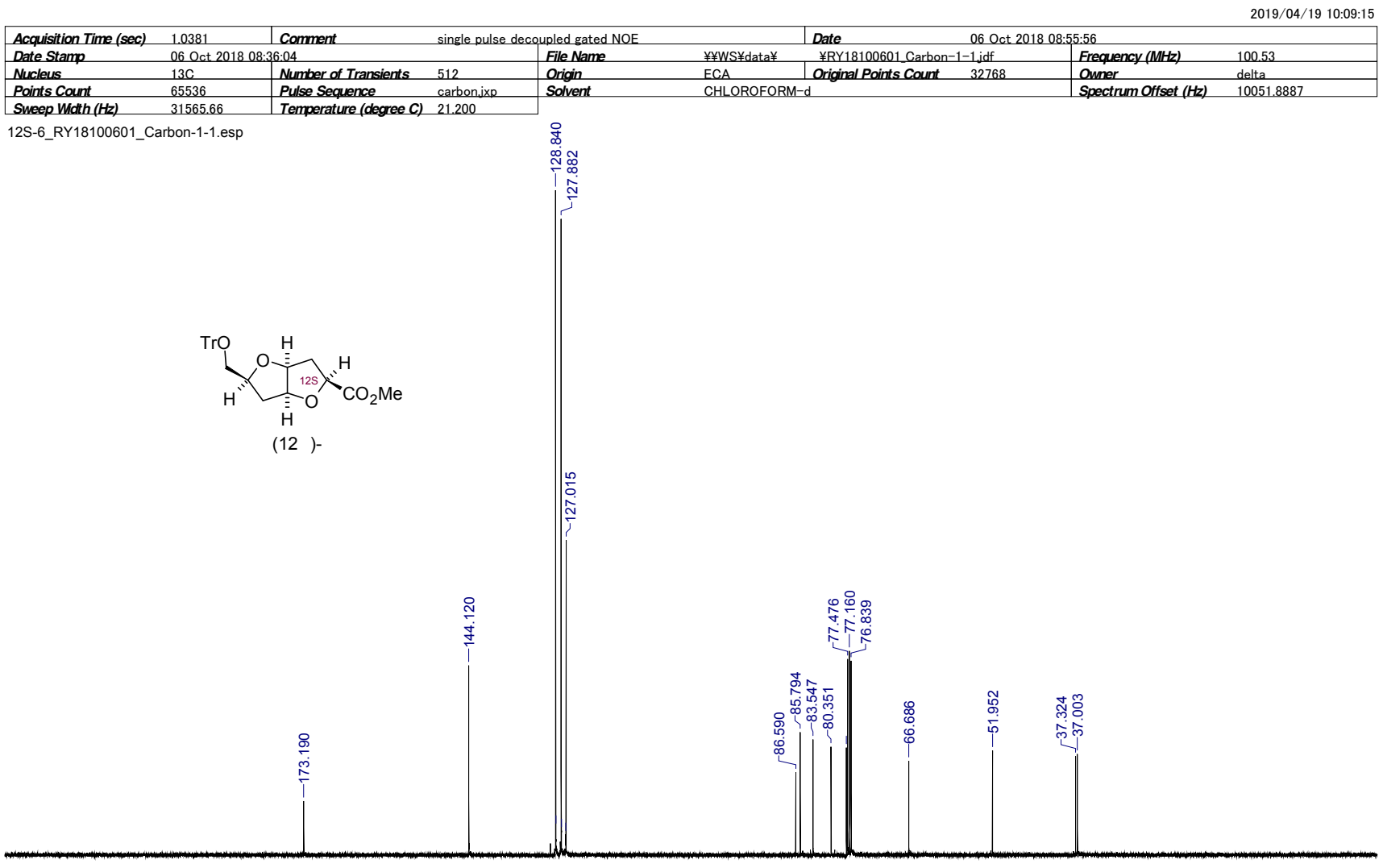

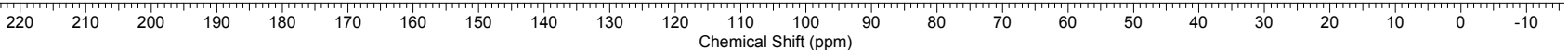


2019/04/19 10:10:12
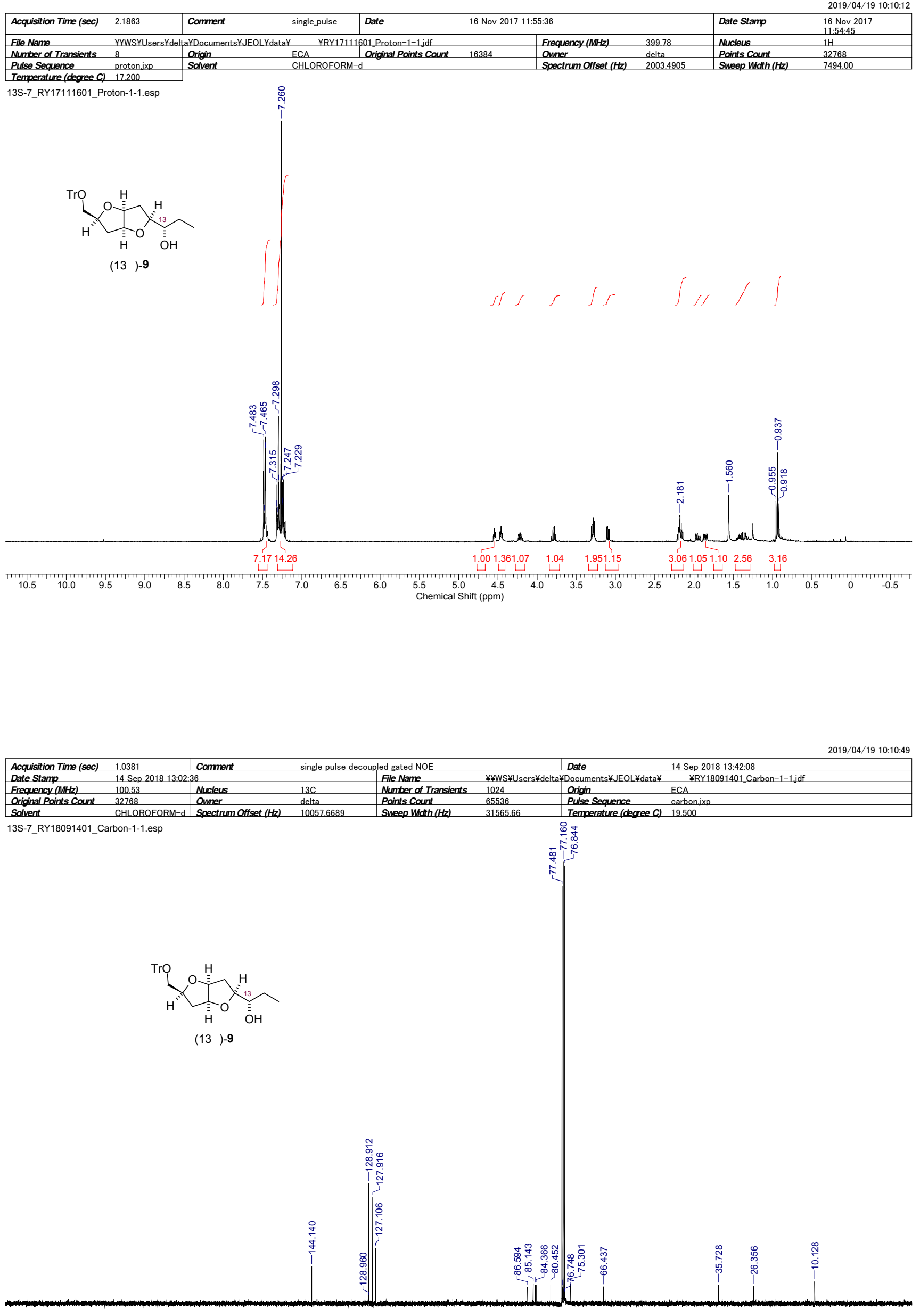

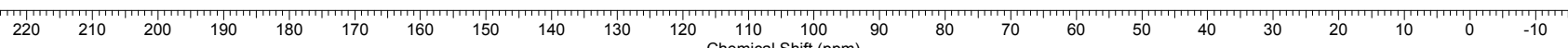


2019/04/19 10:12:36

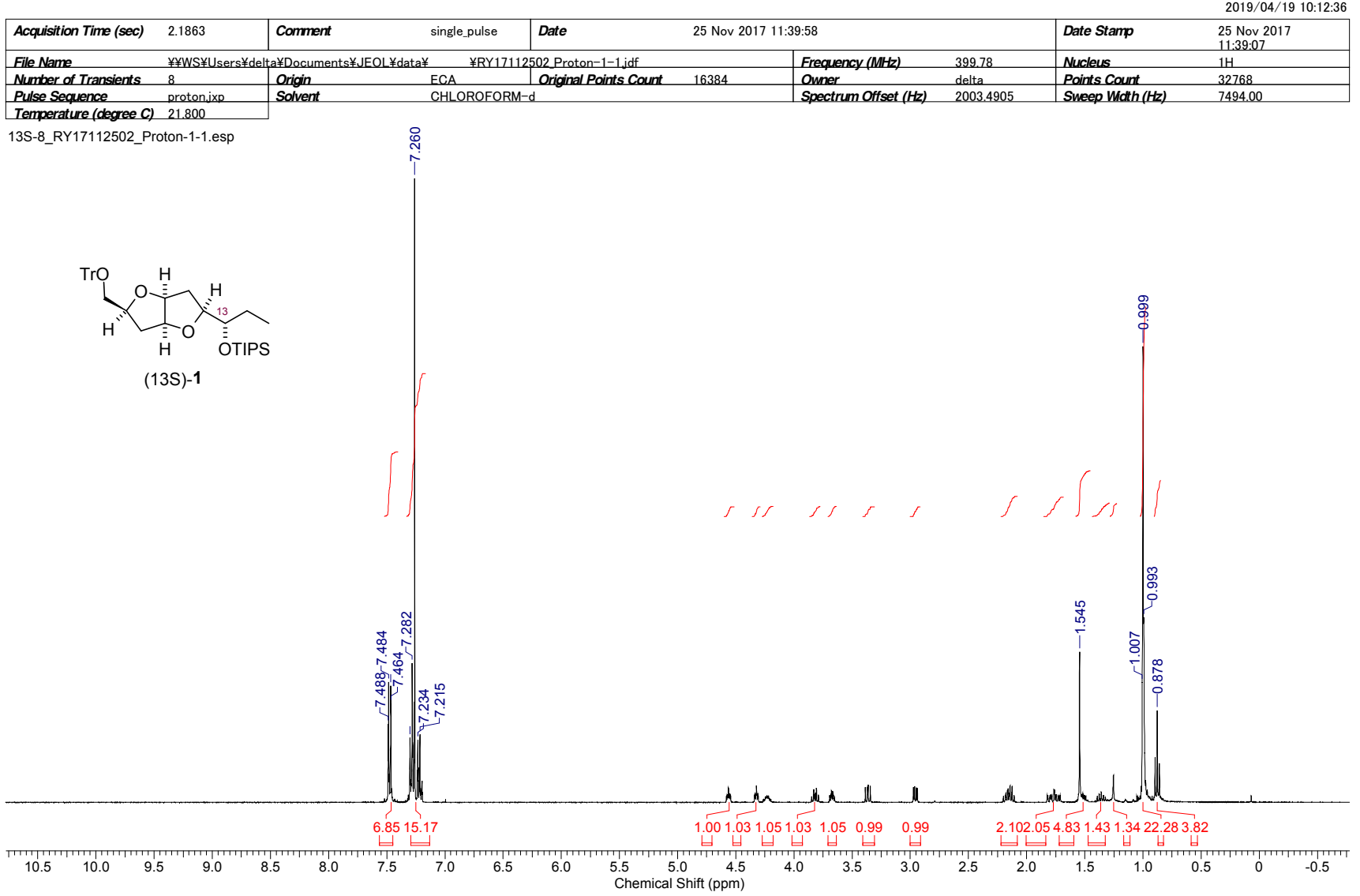

\begin{tabular}{|lll|l|}
\hline Acquisition Time (sec) & 1.0381 & Comment \\
\hline Date Stamp & 28 Aug 2018 09:27:08 \\
\hline Frequency (MHz) & 100.53 & Nucleus \\
\hline Original Points Count & 32768 & Ouner \\
\hline Solvent & CHLOROFORM-d & Spectru \\
\hline 13S-8_RY18082802_Carbon-1-1.esp & \\
\hline
\end{tabular}

(13S)-1

2019/04/19 10:13:05

\begin{tabular}{|ll}
\hline mment & single pulse decoup \\
& \\
\hline cleus & $13 \mathrm{C}$ \\
\hline mer & delta \\
ectrum Offset $(\mathrm{Hz})$ & 10059.1133 \\
\hline
\end{tabular}

File Name

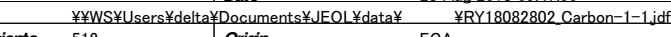

$\begin{array}{llll}\text { Points Count } & 512 & \text { Origin } & \\ & 6536 & \text { Pulse Sequence } & \text { carbon. }\end{array}$

Temperature (degree C) 23.400

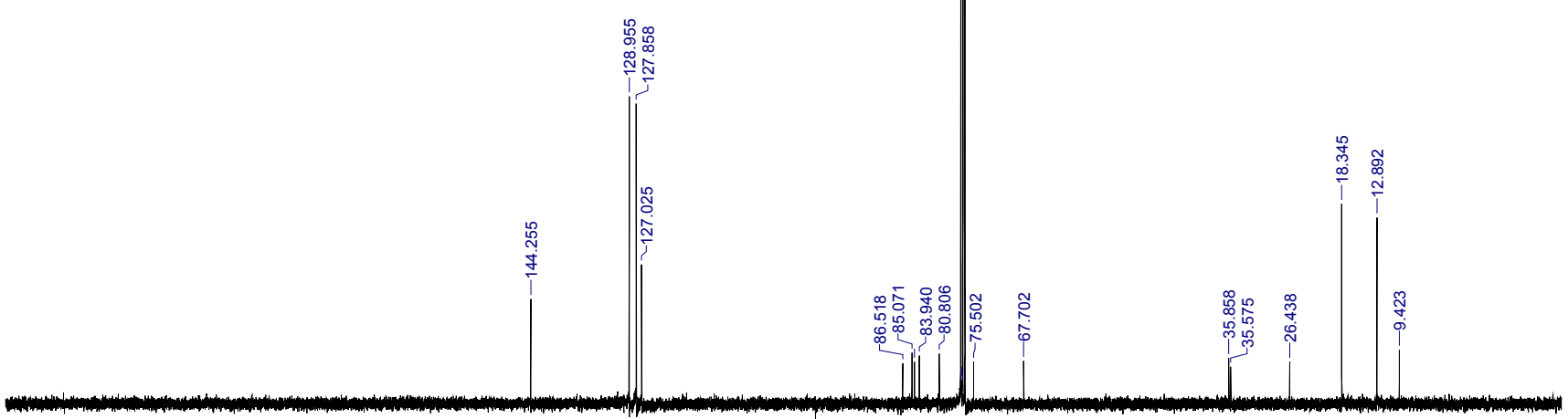

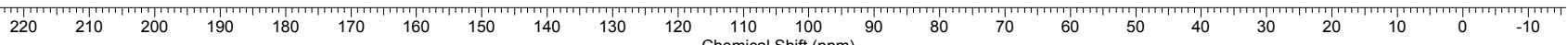



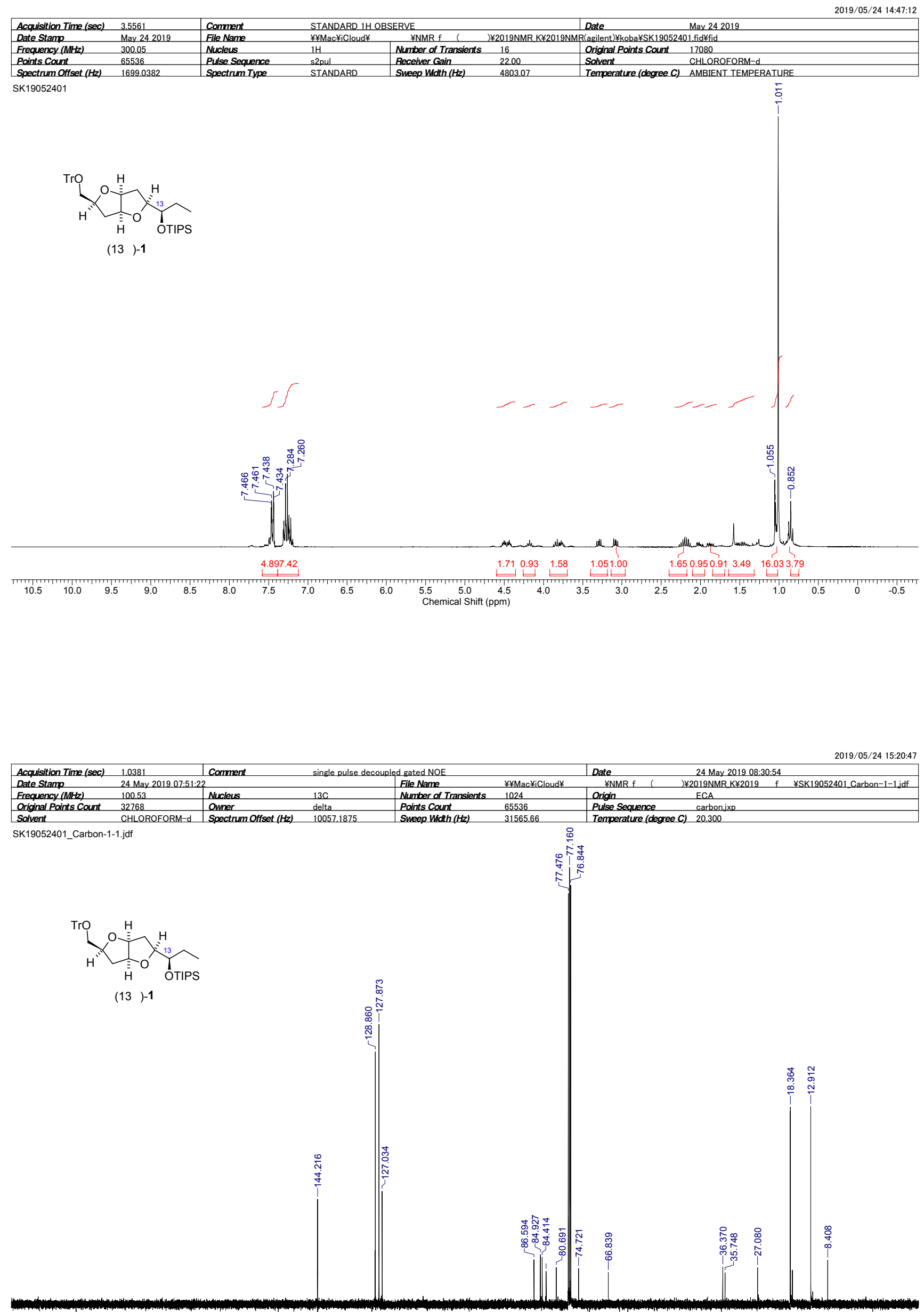

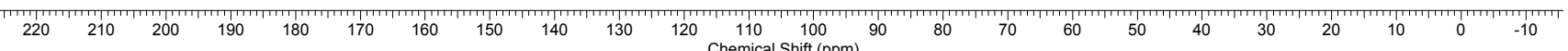


2019/04/19 10:13:56
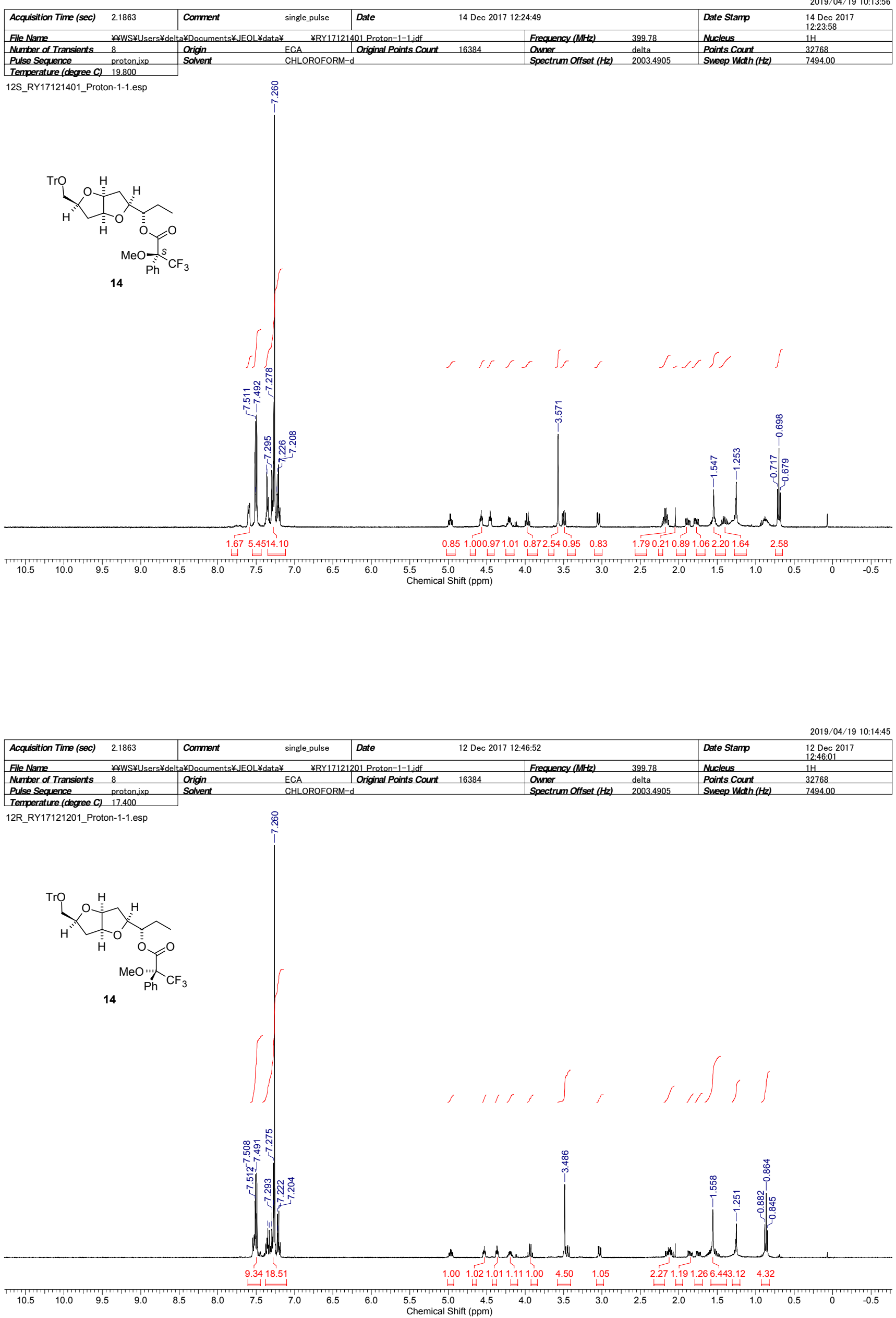
2019/04/19 10:15:37

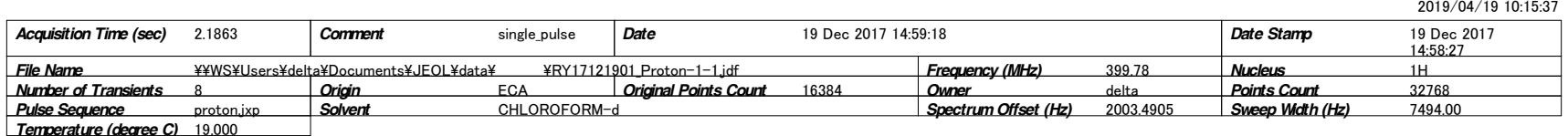

(degree C) 19.000

S3_RY17121901_Proton-1-1.esp

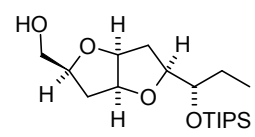

s3

1.00 0.980 .991 .020 .991 .021 .02

\begin{tabular}{|ll|ll|l|l|}
\hline Acquisition Time (sec) & 1.0381 & Comment & \multicolumn{2}{l}{ single pulse decoupled gated } & File \\
\hline Date Stamp & 28 Aug 2018 08:59:41 & Nucleus & 13 C & Num \\
\hline Frequency (MHz) & 100.53 & Ouner & delta & Poirts \\
\hline Original Points Count & 32768 & CHLOROFORM-d & Spectrum Offset (Hz) & 10056.7051 & Swe \\
\hline Solvent & &
\end{tabular}

S3_RY18082801_Carbon-1-1.esp

2019/04/19 10:16:07

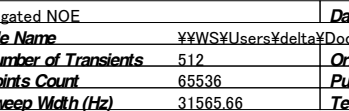

$$
\text { Date }
$$
28 Aug 2018 09:19:33 ¥RY18082801 Carbon-1-1.jdf Pulse Sequence ECA $\begin{array}{ll}\text { Puse } & \text { carbon.jxp } \\ \text { Temperature (degree C) } & 23.300\end{array}$

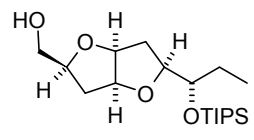

s3

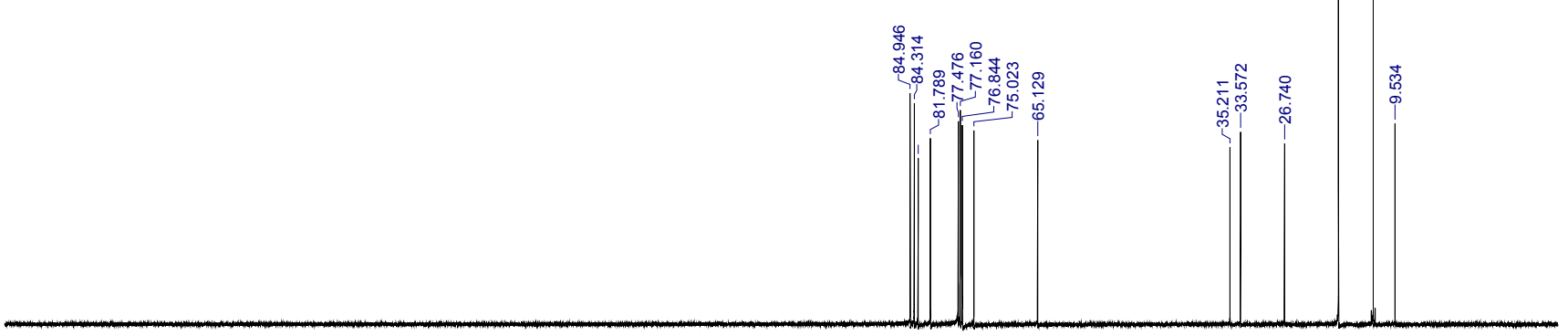


2019/04/19 10:16:45

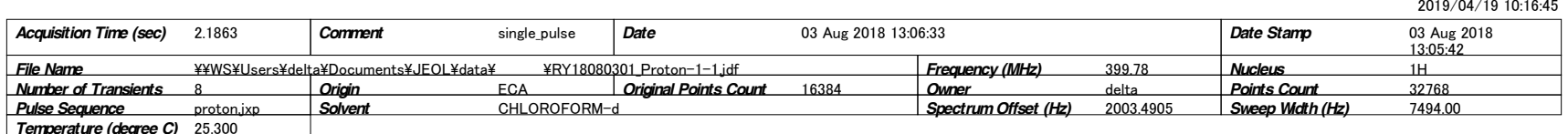

C) 25.300

10_RY18080301_Proton-1-1.esp

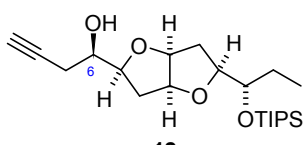

$\stackrel{\stackrel{8}{i}}{i}$

12

his h

0.921 .000 .961 .920 .99

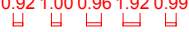

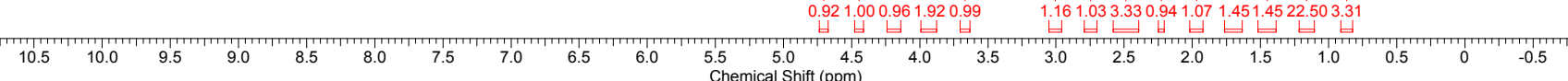

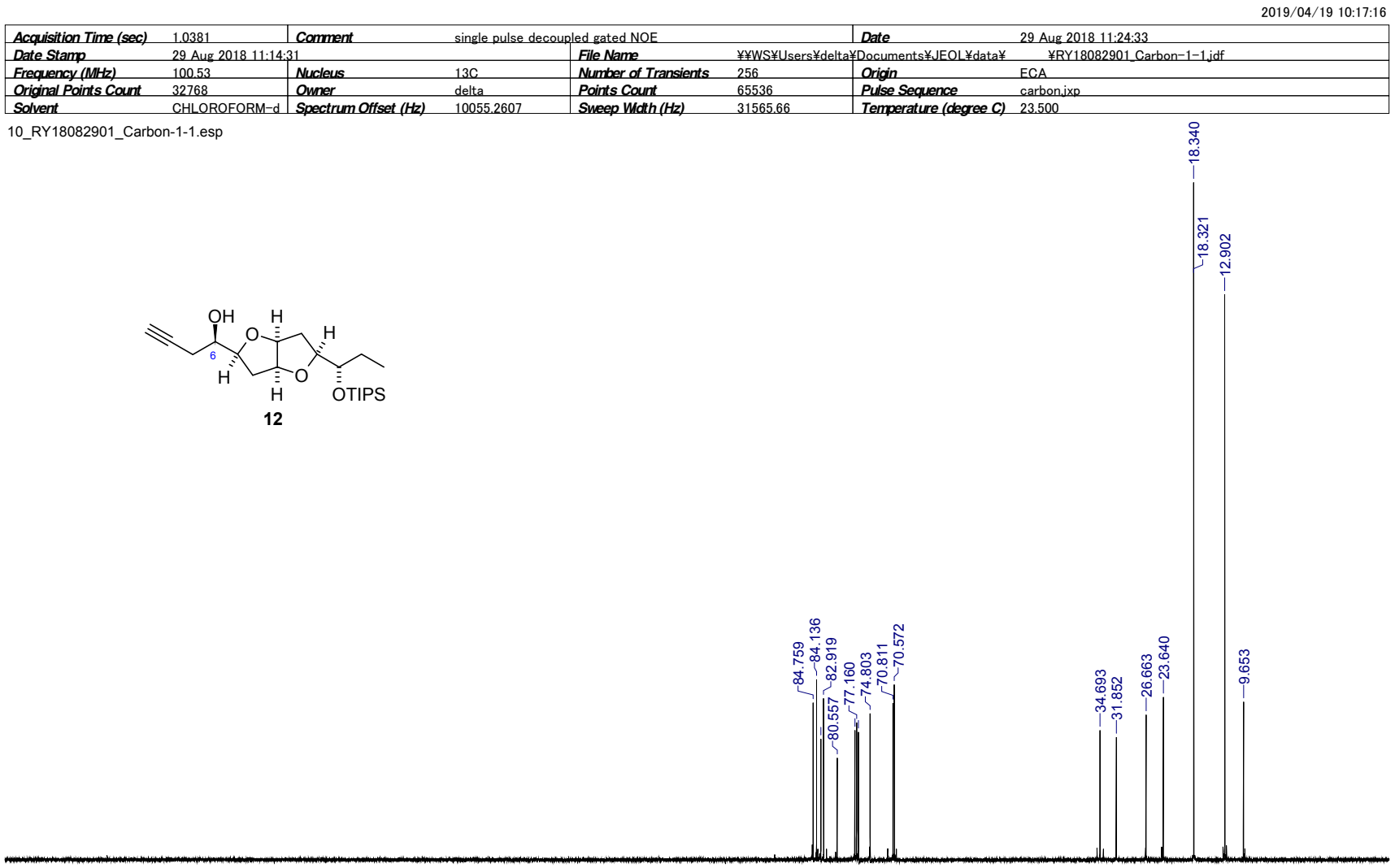

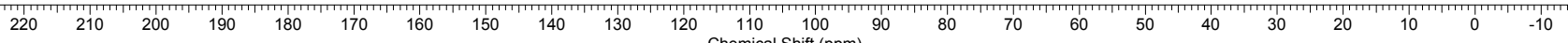




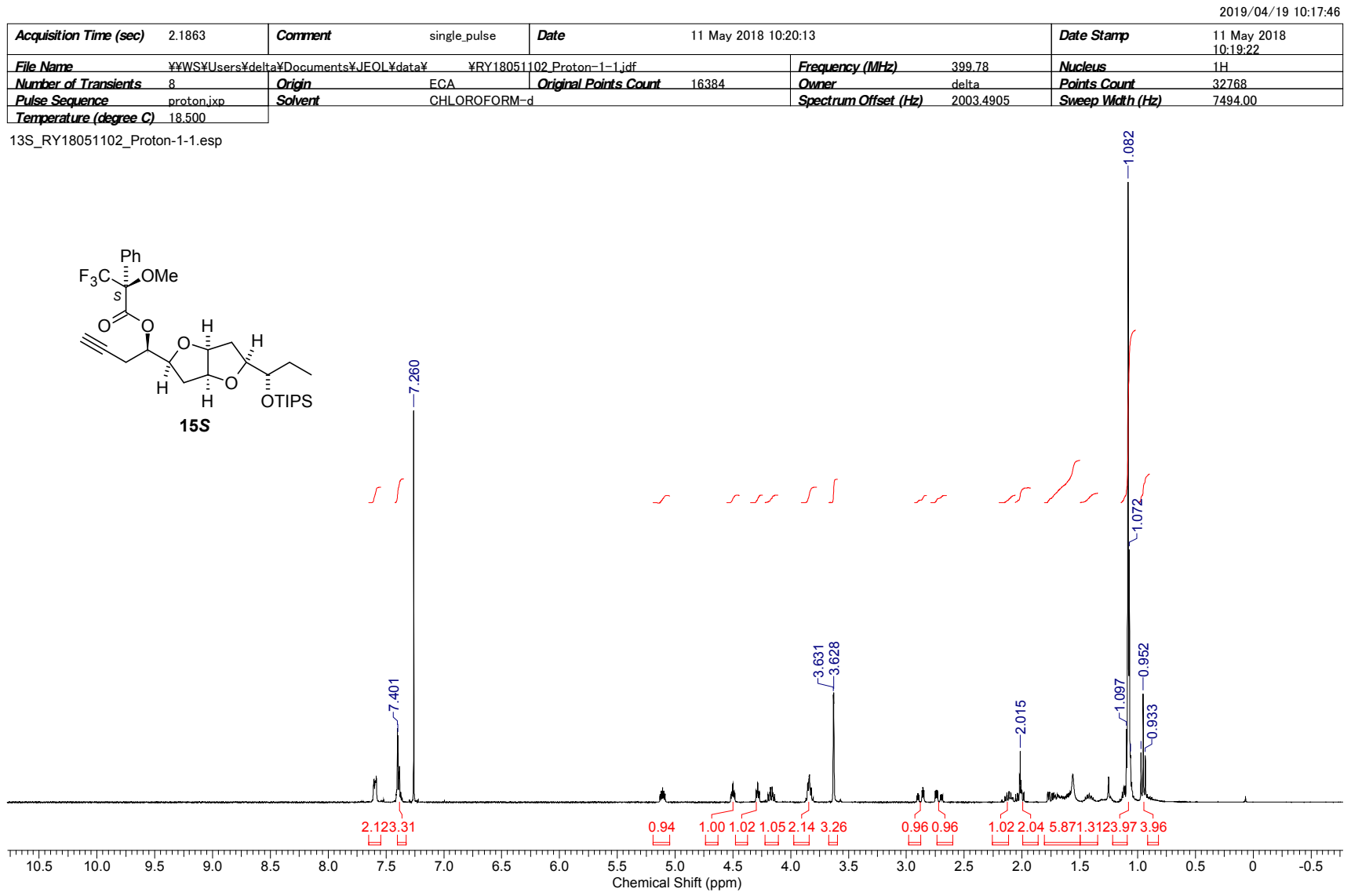

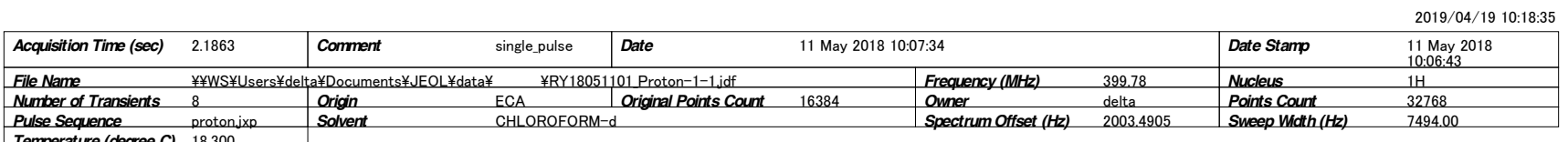

\begin{tabular}{|ll}
\hline Pulse Sequence & proton.jx \\
\hline Temperature (degree C) & 18.300
\end{tabular}

13R_RY18051101_Proton-1-1.esp

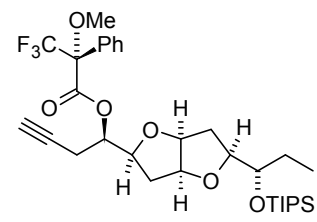

15

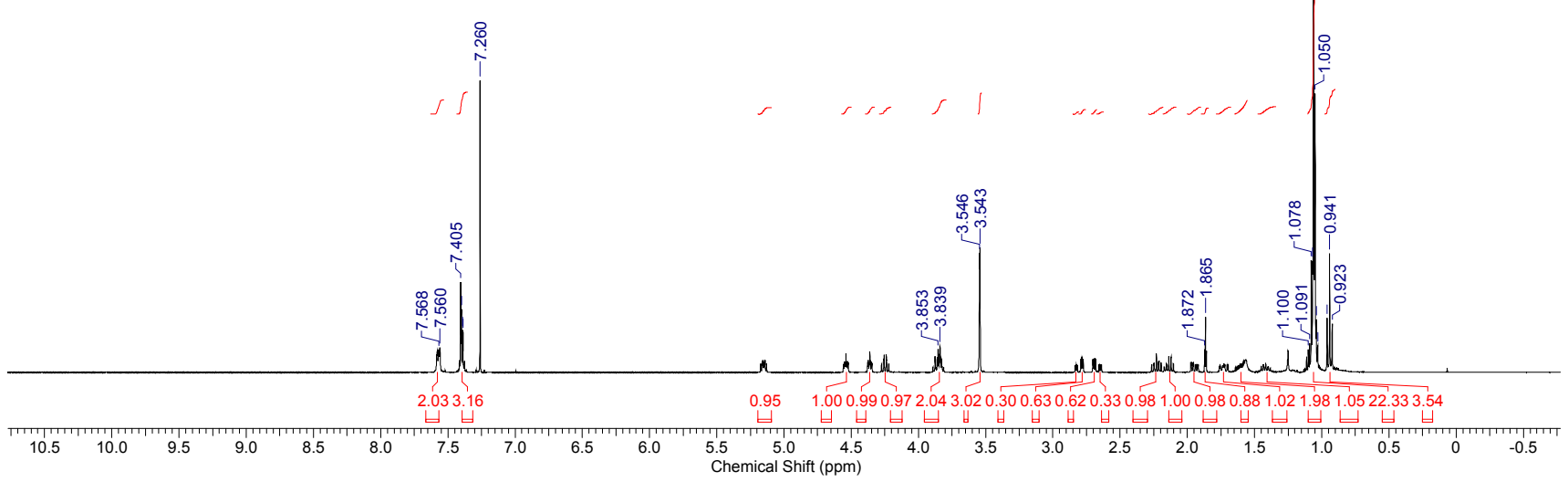


2019/04/19 10:19:15

\begin{tabular}{|c|c|c|c|c|c|c|c|}
\hline Acquisition Time (sec) & 2.1863 & single pulse & Date & 19 Apr 2018 & $88: 57: 02$ & & 19 Apr 2018 08:56:11 \\
\hline File Name & $¥ ¥ W S ¥$ data $¥$ & \#RY18041901 Proton-1-1.jdf & Frequency $(\mathrm{MHz})$ & 399.78 & Nucleus & $1 \mathrm{H}$ & Number of Transients \\
\hline Origin & ECA & Original Points Count & Ouner & delta & Points Count & 32768 & Pulse Sequence \\
\hline Solvent & CHLOROFOP & & Spectrum Offset ( $\mathrm{Hz}_{2}$ & 2003.4905 & Sweep Width $(\mathrm{Hz})$ & 7494.00 & Temperature (degree C) 18.300 \\
\hline
\end{tabular}

Solvent CHLOROFORM-d
11_RY18041901_Proton-1-1.esp

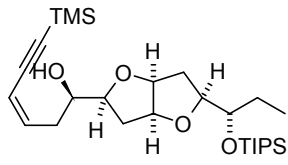

13

OTIPS

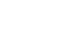

.

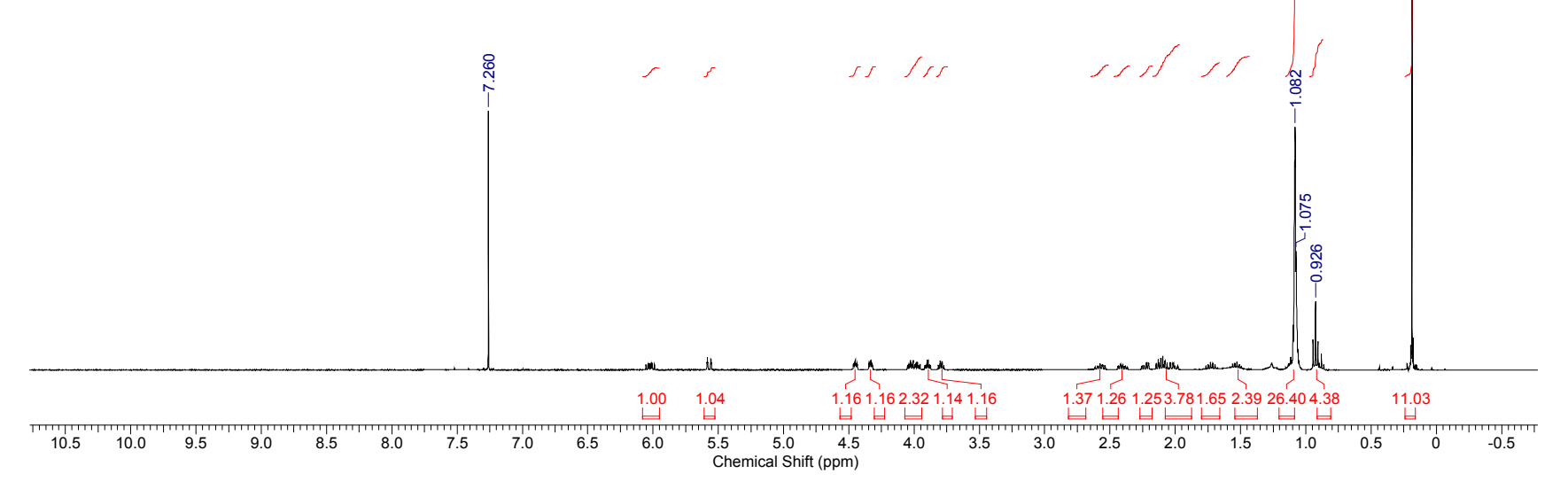

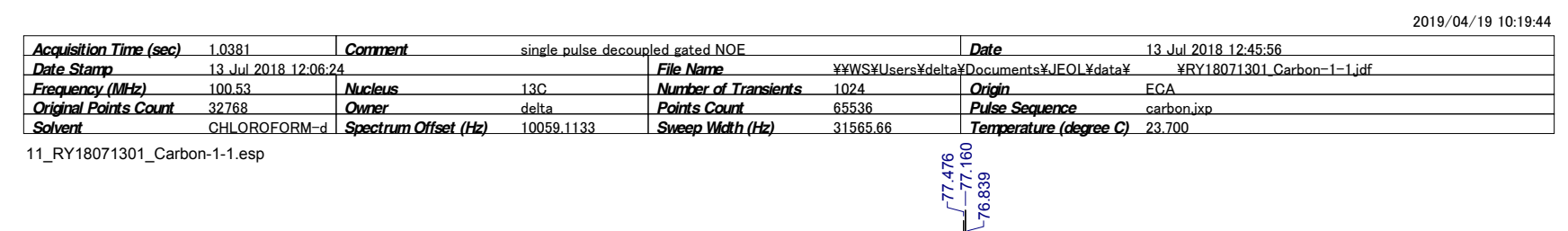

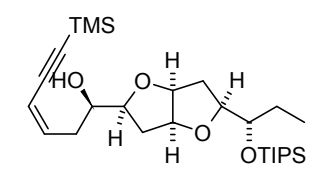

13

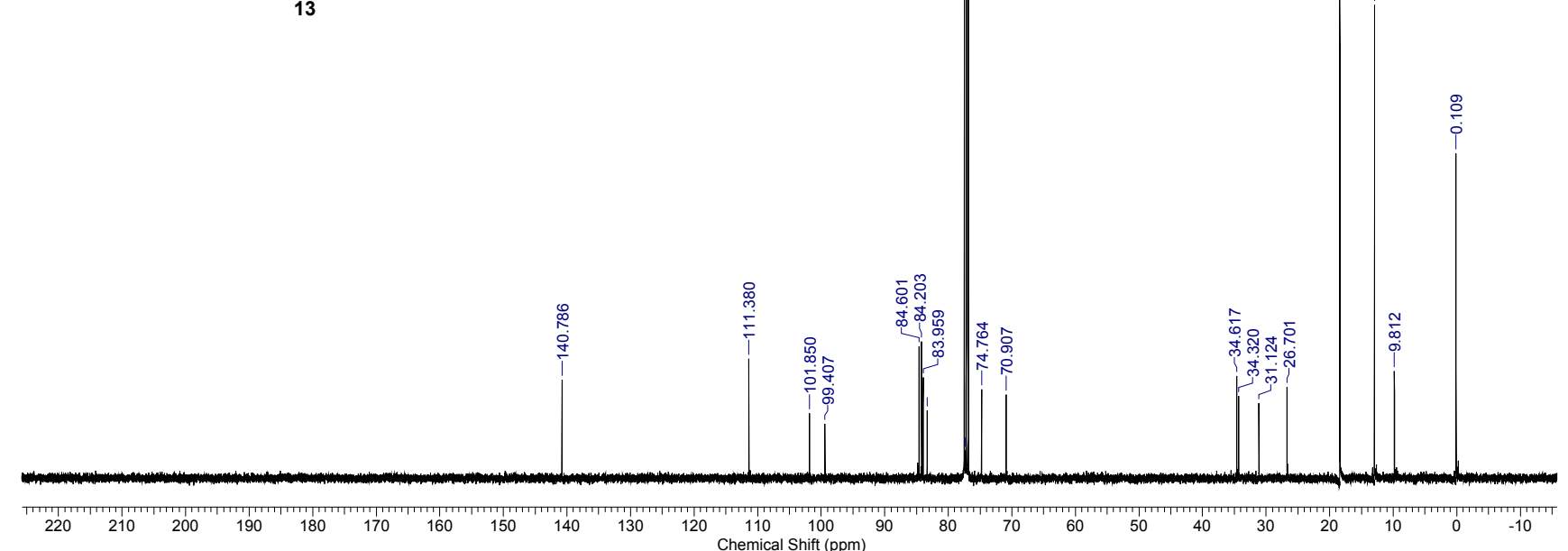


2019/04/19 10:20:15

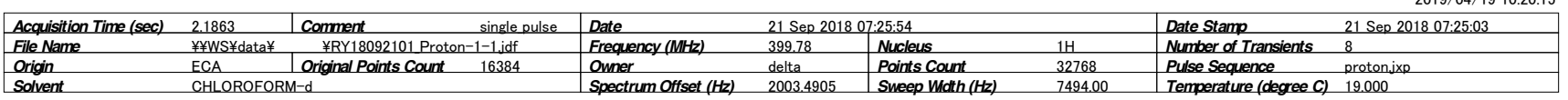

\begin{tabular}{|l|ll|llll}
\hline Solvent $\quad$ CHLOROFORM-d & Spectrum Offset (Hz) & 2003.4905 & Sweep Width (Hz) & 7494.00 & Temperature (degree C) & 19.000 \\
S4_RY18092101_Proton-1-1.esp & & &
\end{tabular}

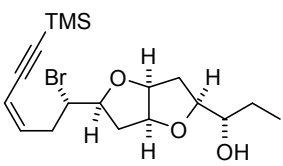

S4
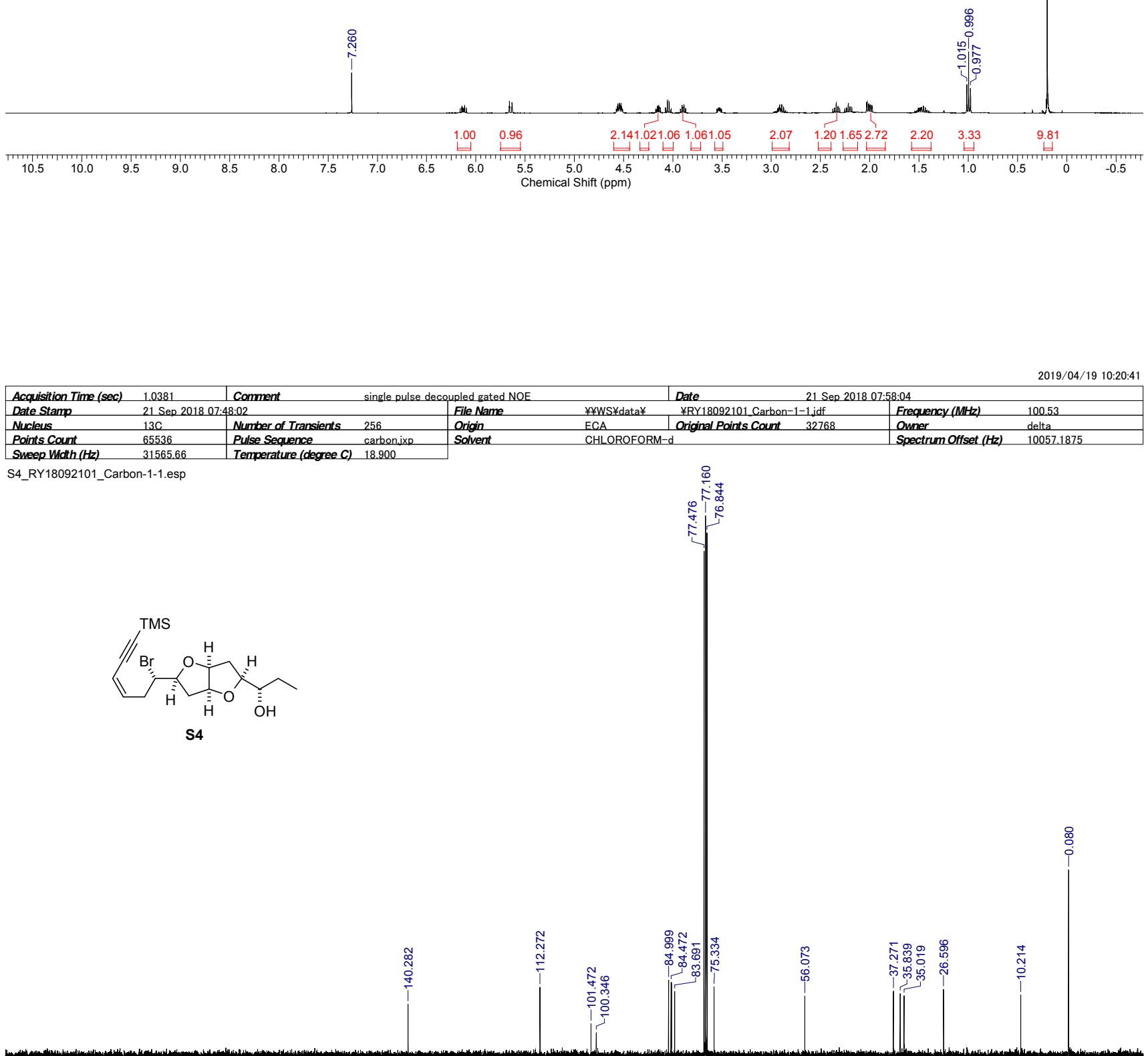

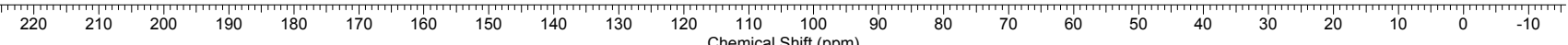


2019/04/19 10:21:17

\begin{tabular}{|c|c|c|c|c|c|c|c|c|}
\hline Acquisition Time (sec) & 2.1863 & Cormment & single_pulse & \multicolumn{3}{|c|}{16 Oct 2018 11:21:29 } & Date Stamp & $\begin{array}{l}\text { 16 Oct } 2018 \\
11: 20: 38\end{array}$ \\
\hline File Name & $¥ \#$ WS $\# U$ & a¥Documen & \#RY18 & 601 Proton-1-1.jdf & Frequency $(\mathrm{MHz})$ & 399.78 & Nucleus & $1 \mathrm{H}$ \\
\hline Number of Transients & 8 & Origin & ECA & Original Points Count & Ouner & delta & Points Count & 32768 \\
\hline Pulse Sequence & proton.jx & Solvent & CHLOROFOF & & Spectrum Offset (Hz) & 2003.4905 & Sweep Width $(\mathrm{Hz})$ & 7494.00 \\
\hline
\end{tabular}

Terperature (degree C) 18.700

1_RY18101601_Proton-1-1.esp

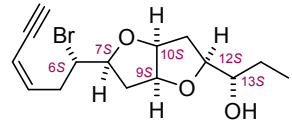

(6S, 7S,9S, 10S, 12S, 13S)-1

(+)-isolaurenidificin

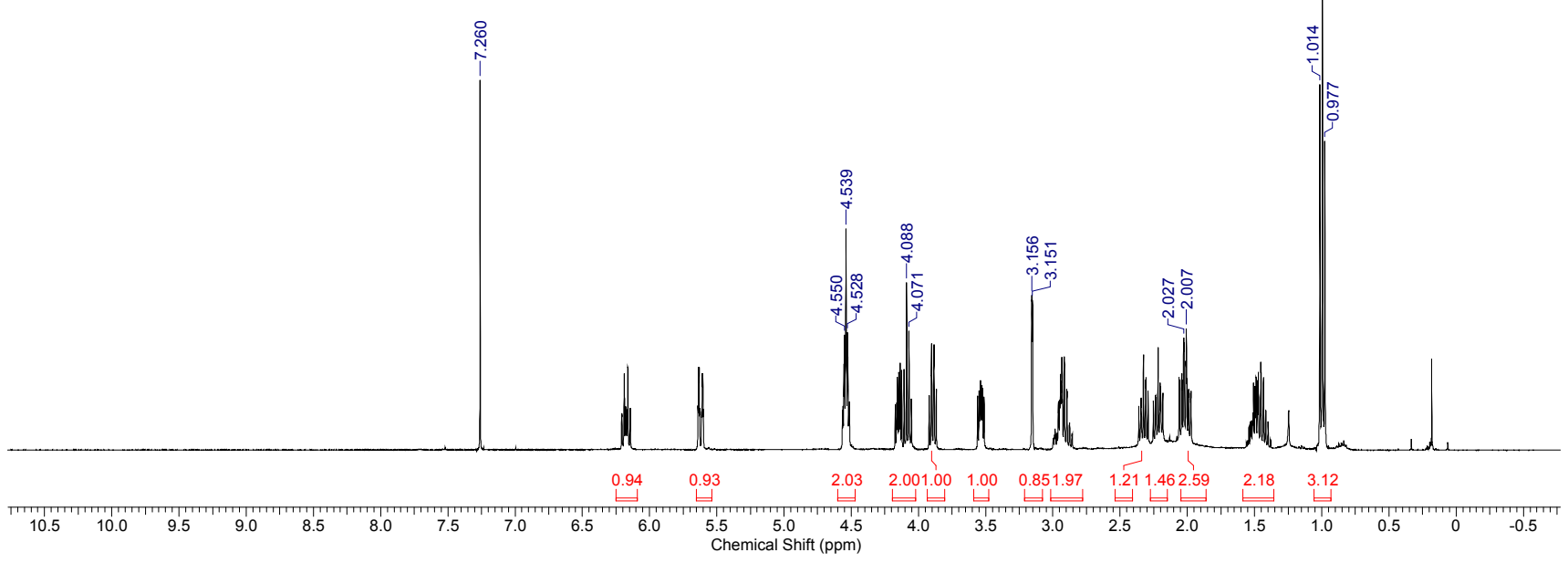

\begin{tabular}{|c|c|c|c|c|c|c|c|c|c|}
\hline Acquisition Time (sec) & 2.1863 & Comment & single_pulse & Date & \multicolumn{3}{|c|}{16 Oct 2018 11:21:29 } & Date Stamp & $\begin{array}{l}16 \text { Oct } 2018 \\
11: 20: 38\end{array}$ \\
\hline File Name & \multirow{2}{*}{\multicolumn{3}{|c|}{ 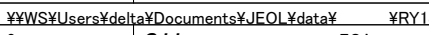 }} & 601 Proton-1-1.jdf & & Frequency (MHz) & 399.78 & Nucleus & $1 \mathrm{H}$ \\
\hline Number of Transients & 8 & & \multirow{2}{*}{\multicolumn{2}{|c|}{\begin{tabular}{l|l} 
ECA & Original Points Count \\
CHLOROFORM-d
\end{tabular}}} & 16384 & Owner & delta & Points Count & 32768 \\
\hline Pulse Sequence & proton.jx & Solvent & & & & Spectrum Offset (Hz) & 2003.4905 & Sweep Width $(\mathrm{Hz})$ & 7494.00 \\
\hline
\end{tabular}

Temperature (degree C) 18.700

1_RY18101601_Proton-1-1.esp

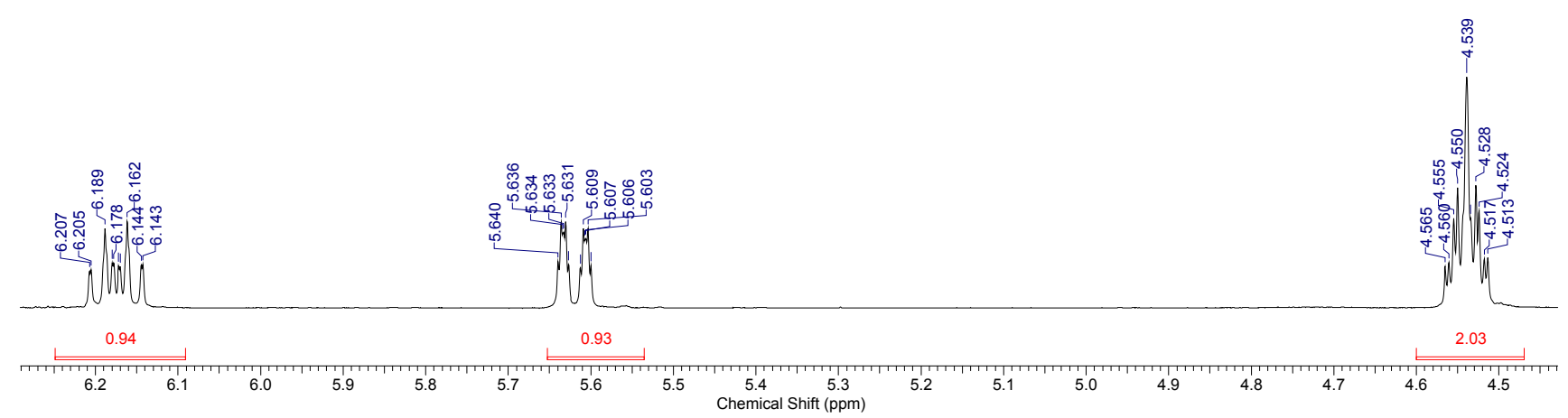


2019/05/09 20:29:45

\begin{tabular}{|c|c|c|c|c|c|c|c|c|}
\hline Acquisition Time (sec) & 2.1863 & Comment & single_pulse & \multicolumn{3}{|c|}{16 Oct 2018 11:21:29 } & Date Stamp & 16 Oct 2018 \\
\hline File Name & \multicolumn{3}{|c|}{ 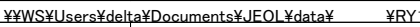 } & 601 Proton-1-1.jdf & Frequency (MHz) & 399.78 & Nucleus & $1 \mathrm{H}$ \\
\hline Number of Transients & 8 & Origin & ECA & Original Points Count & Ouner & delta & Points Count & 32768 \\
\hline Pulse Sequence & proton.jx & Solvent & CHLOROFO & & Spectrum Offset $(\mathrm{Hz}$ & 2003.4905 & Sweep Width $(\mathrm{Hz})$ & 7494.00 \\
\hline
\end{tabular}

Temperature (degree C) 18.700

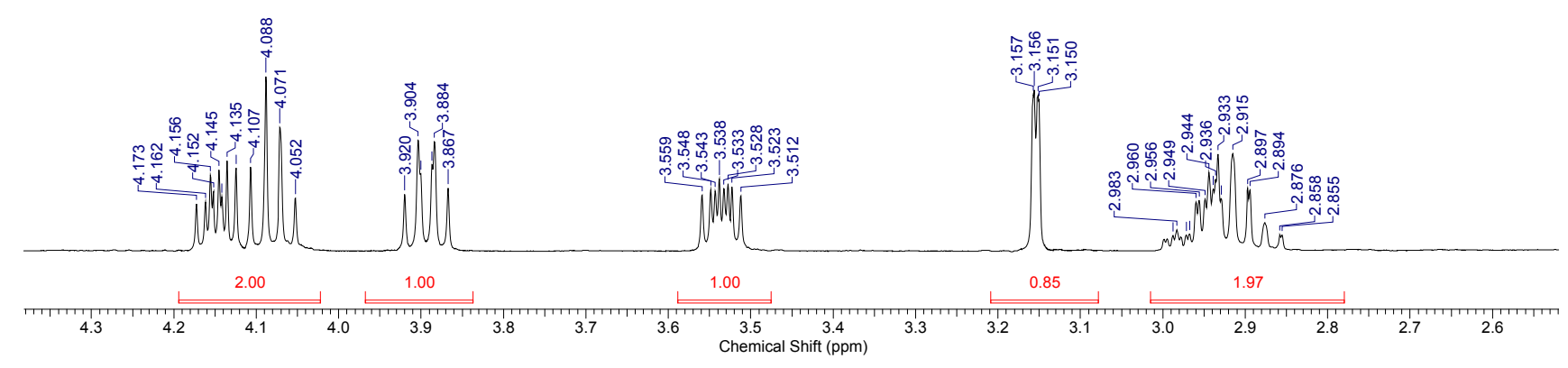

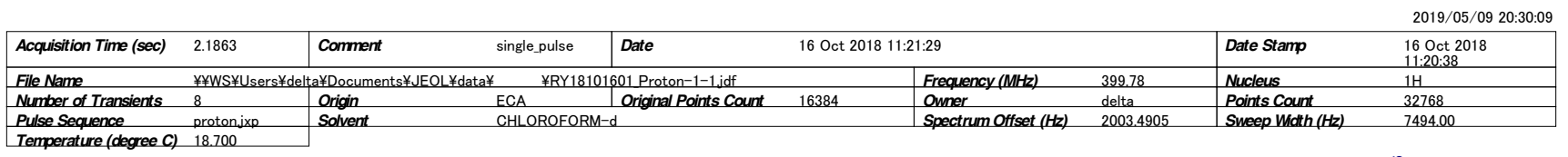

Temperature (degree C) 18.700

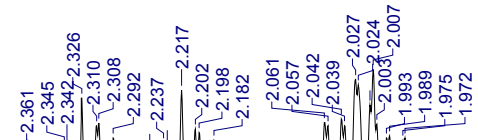

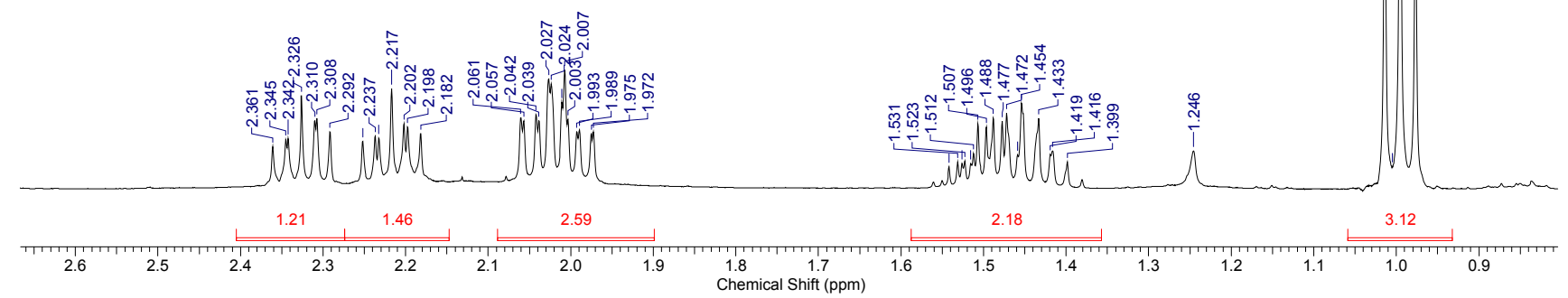

Sweep Width $(H$ 
2019/04/19 10:21:52

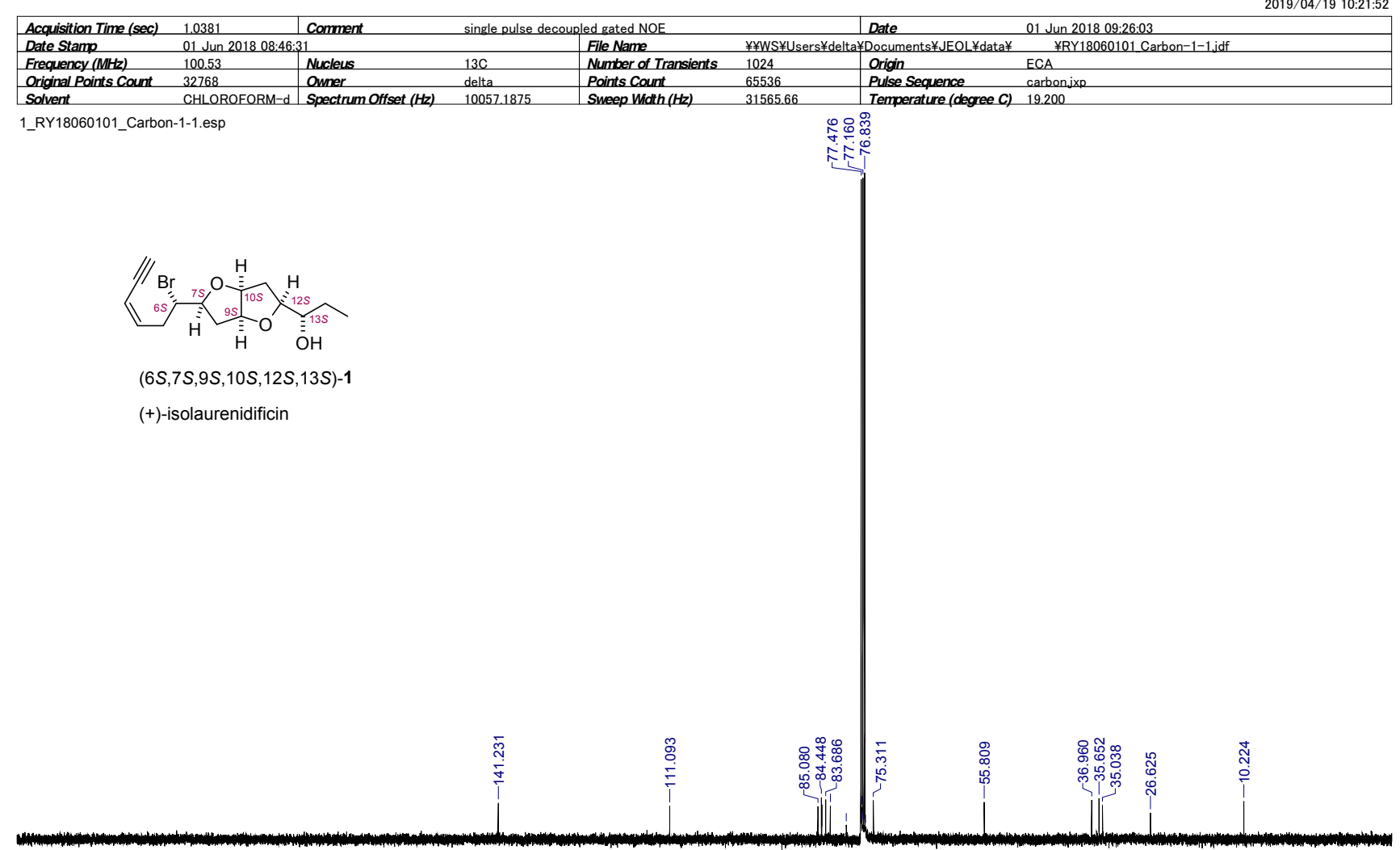

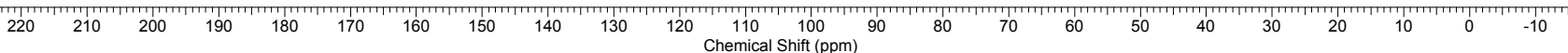

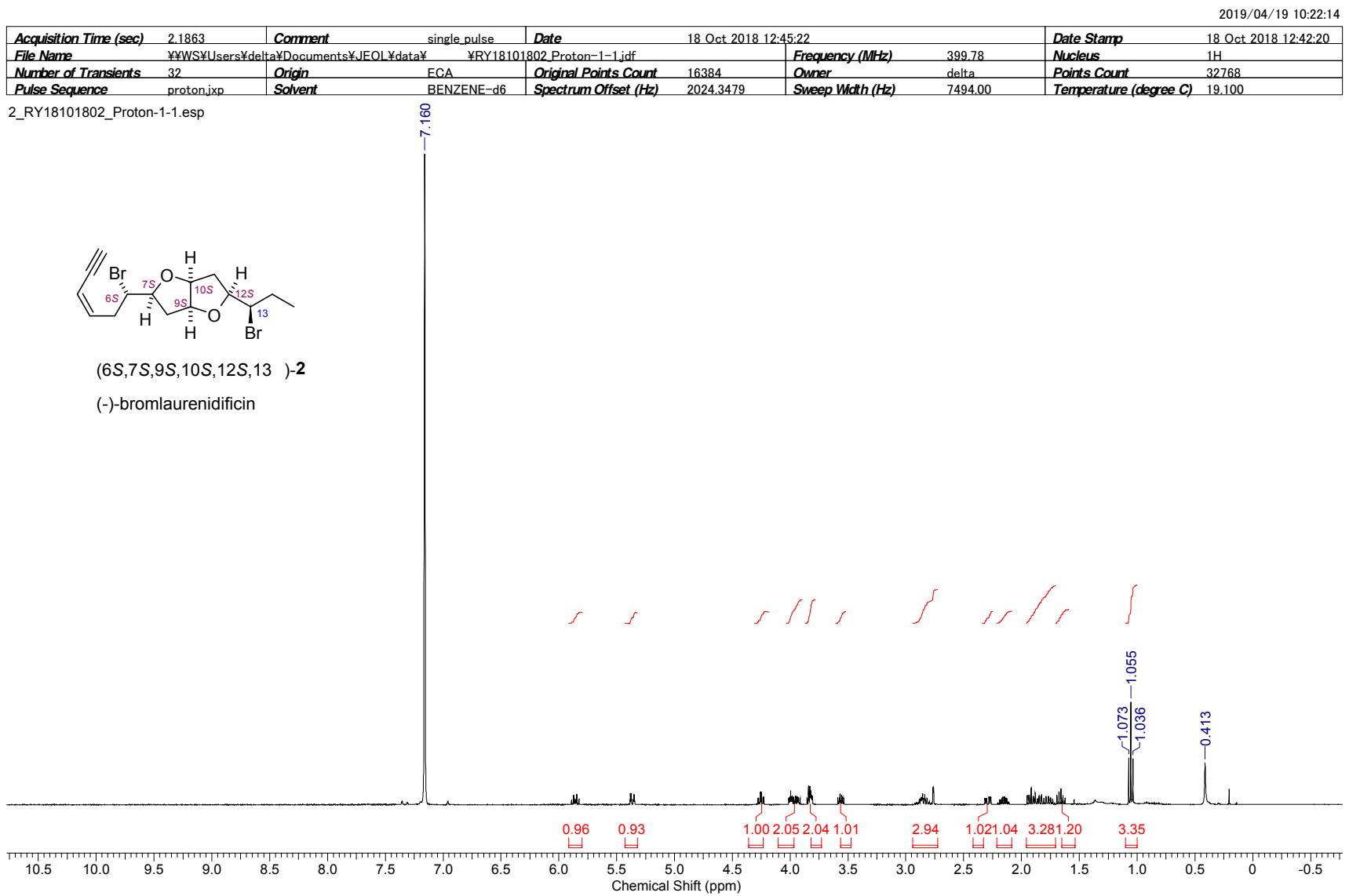


2019/05/09 20:31:37

\begin{tabular}{|c|c|c|c|c|c|c|}
\hline & & & & & & \multirow[b]{2}{*}{$\mathrm{IH}$} \\
\hline & & & & & & \\
\hline
\end{tabular}

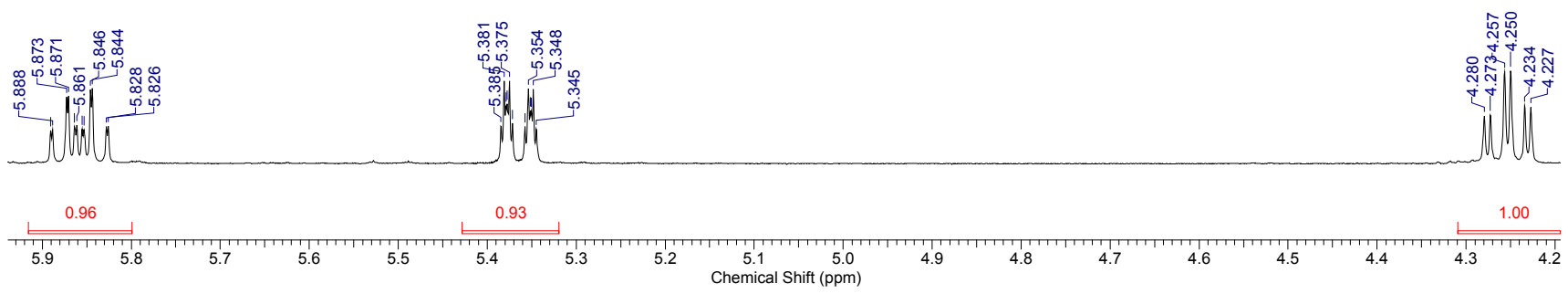

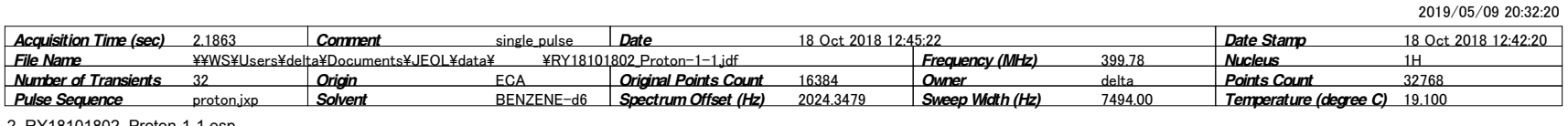

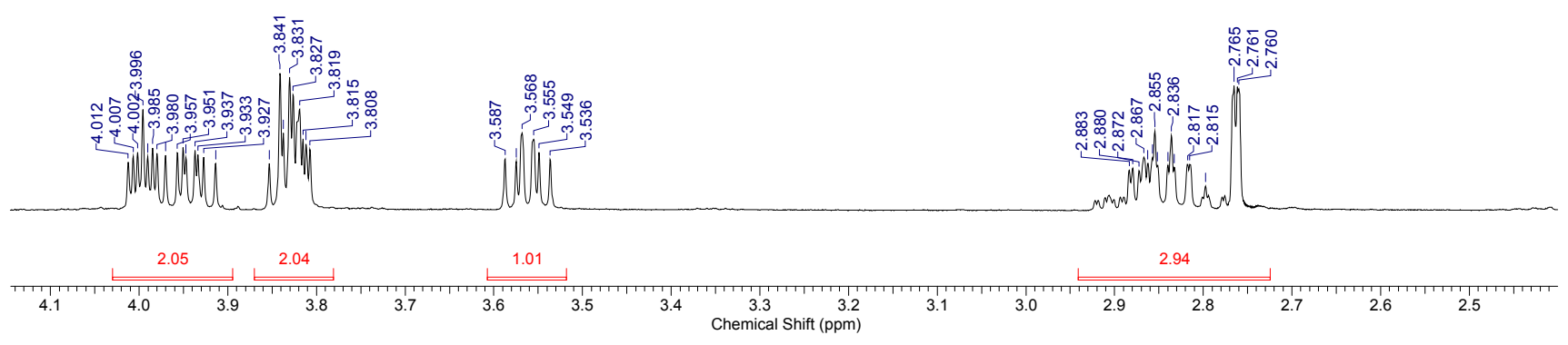



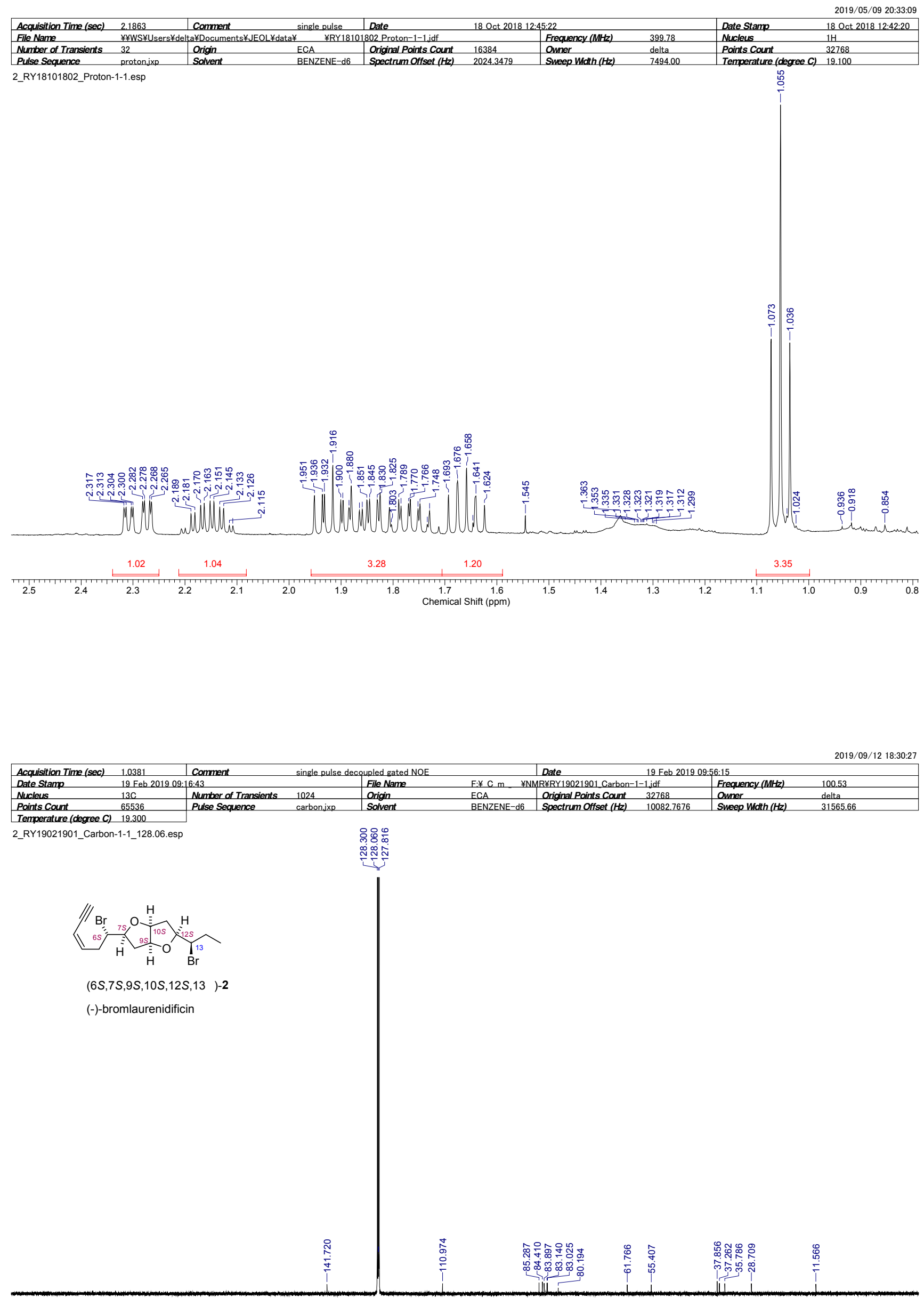

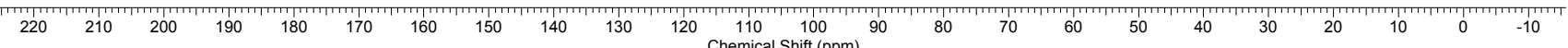

